\title{
Reducción de la jornada laboral en un contexto de producción en el hogar
}

\author{
José Ramos* \\ Universidad Europea de Madrid \\ jose.ramos@uem.es
}

\begin{abstract}
RESUMEN
Este trabajo se centra en analizar la reducción de la jornada laboral no sólo como una política de creación de empleo, sino como una política social que puede mejorar el bienestar de los individuos mediante una mejor conciliación de la vida familiar y laboral. Partiendo de un modelo de emparejamientos donde se introduce producción en el hogar, la reducción de jornada se vislumbra como una medida que destruye más empleo y que provoca una reducción salarial mayor de lo que se estaba considerando en los modelos anteriormente desarrollados. No obstante, reducir el tiempo destinado a actividades de mercado, puede permitir una mayor conciliación con la vida familiar, lo cual puede acarrear un incremento del nivel de utilidad del individuo que compensa la tradicional reducción vía empleo y salario.
\end{abstract}

* Agradezco la colaboración de Maite Blázquez de la Universidad Autónoma de Madrid. Así mismo agradezo a los participantes en el XXXI Simposio sobre Análisis Económico por sus comentarios y sugerencias. 
Clasificación JEL: J20, J21, J22, J29, J38, J58.

Palabras Clave: Reducción de Jornada, Producción en el Hogar, Políticas Laborales, Creación de Empleo, Conciliación Familiar.

\section{INTRODUCCIÓN}

El problema desempleo se ha convertido en uno de los problemas económicos que más preocupan a los ciudadanos de la vieja Europa. A lo largo del tiempo la mayoría de los países han planteado, con mayor o menor éxito, distintas políticas con objeto de incrementar el nivel de empleo, como por ejemplo las destinadas a flexibilizar las condiciones de contratación y despido. Si nos fijamos detenidamente en la economía española vemos que la preocupación por el desempleo ha sido historicamente de especial relevancia. En particular, el mercado laboral español se ha enfrentado a una de las mayores tasas de desempleo en el conjunto de la OECD, desde mediados de los $80^{1}$.

El objeto del presente trabajo se centra en analizar una de las medidas de política económica que mayor controversia ha provocado en las últimas décadas: la reducción de la jornada laboral. Las políticas de reducción de jornada se han implementado con mayor o menor fortuna. En este sentido Francia ha sido uno de los países pioneros en la reducción de su jornada laboral, pasando de 40 horas semanales a 39 horas, para finalmente aprobar una reducción de jornada a 35 horas semanales ${ }^{2}$. No obstante, los resultados consecuencia de esta la política tanto en Francia como en otros países europeos, como Alemania y Holanda, que han seguido esta misma senda, no han sido demasiado positivos. Esto ha llevado a la mayoría de gobiernos, que habían considerado esta medida como una solución para sus problemas de desempleo, cuestionarse su validez y replantear la estrategia. En España la reducción de jornada se ha planteado como una antigua reivindicación por los sindicatos de nuestro

${ }^{1}$ Incluso en el año 2000 la tasa de paro en España según datos de Eurostat (2003) alcanzaba el $11.3 \%$ frente al $7.8 \%$ de la media de la UE15.

${ }^{2}$ El 13 de Junio de 1998 se aprueba en Francia por el gobierno de Jospin la ley que establece la reducción de jornada de 39 a 35 horas. Dicha norma entraría en vigor el 1 de Enero de 2000 para todas las empresas con más de 20 trabajadores y el 1 de Enero de 2002 para el resto. 
país con el objetivo claro de reducir el desempleo. Sin embargo, al margen de considerar la reducción de jornada como una política de reducción de desempleo, cada vez más se ha planteado esta medida como una política social. En los últimos años se ha abierto el debate en las economías más desarrolladas, y en especial en la Europa del bienestar, sobre la necesidad de incentivar aquellas políticas que consigan reconciliar la vida laboral con la vida familiar. Siguiendo con este enfoque, la reducción de jornada laboral no debe verse sólo como una política de reparto de empleo (tal y como fué concebida en su origen), sino también como una medida con un fuerte contenido social, y por tanto, donde es esencial valorar el bienestar de los ciudadanos. Este segundo enfoque, centrado en el bienestar, ha sido escasamente estudiado en la literatura.

El principal objeto de este trabajo es analizar las implicaciones económicas de una reducción de jornada, no sólo vista como una medida de reparto de empleo, sino considerándola una medida que puede mejorar el bienestar de los ciudadanos al conseguir mejorar la conciliación del tiempo dedicado a la producción de mercado y a la producción en el hogar, en línea con las mayoría de las políticas sociales que se reivindican en la Europa comunitaria. La cuestión que se plantea en el presente trabajo es si efectivamente, dicha medida es eficiente para la economía, y si además lleva a una mejora del bienestar de los hogares. Para dar respuesta esta pregunta el marco de análisis que se utilizará será un modelo de equilibrio general que incorpore la producción en el hogar como elemento relevante.

El aspecto diferenciador de este trabajo es introducir elementos de la oferta de trabajo en el análisis del impacto de una política de reducción de jornada. La mayoría de los trabajos que se han desarrollado hasta ahora, analizan esta medida laboral desde su vertiente de demanda, como los de Calmorfos y Hoel (1988) y Booth y Schiantearelli (1987). Si bien algunos trabajos recientes como los de Fitzgerald (1996) y Kydland (1995) han puesto de manifiesto la importancia de considerar también los factores de oferta. En esta misma línea, el presente trabajo incorpora elementos de la oferta de trabajo que nos permitan vislumbrar los efectos de la reducción de jornada en dos aspectos, los concernientes al mercado de trabajo y el conjunto de la economía, y aquellos referidos al bienestar de los individuos. En concreto, como hemos señalado, se considerará la producción en el hogar como elemento clave en el análisis de la reducción de jornada. En este sentido, se asume que los individuos pueden dedicar parte de su tiempo disponible a tareas del hogar. Bajo este enfoque se trata de analizar cómo afecta una medida de reducción de jornada sobre la oferta de trabajo de los individuos, así como su impacto, no sólo a nivel macroeconómico, sino también desde el punto de vista del bienestar del individuo. 
Una vez detallado el enfoque del análisis, y puesta de manifiesto la importancia de considerar las actividades del hogar a la hora de estudiar las implicaciones económicas de una reducción de jornada, se plantean varias cuestiones que se pretenden despejar: ¿Cómo repercute la política de reducción de jornada en el tiempo destinado a actividades de mercado y no mercado?, ¿y a su nivel de productividad?, ¿cómo afectará la reducción de la jornada a la producción en el hogar y a la producción en el mercado?, ¿que efecto tendrá esta política sobre el bienestar del individuo en este contexto?,¡se podrá justificar la reducción de jornada como una medida de creación de empleo?, ¿y cómo una medida de mejora del bienestar del individuo?, ¿es realmente una medida que incentive la reconciliación de la vida laboral y familiar?

El trabajo se distribuye de la siguiente manera. En la siguiente sección se lleva a cabo una revisión de los principales trabajos relacionados con la reducción de jornada y la producción en el hogar. En la Sección 3 se desarrolla el marco teórico que se tomará como punto de partida. La Sección 4 se centra en la reducción de jornada con perfecta sustitución y sin producción en el hogar, mientras que en la Sección 5 se analiza el caso de reducción de jornada con producción en el hogar y perfecta sustitución horas-hombre. Finalmente en la Sección 6 se presentan las principales conclusiones.

\section{Literatura Previa}

La idea de la reducción de la jornada laboral como mecanismo de reparto de empleo surgió en la década de los 80 . Los primeros trabajos al respecto, centrados en una visión macroeconómica, se basan en la óptica de equilibrio parcial. En estos trabajos se habla por primera vez de la reducción de la jornada laboral como mecanismo de creación y reparto de empleo. Todos estos trabajos presentan una serie de características comunes. En primer lugar, el agente esencial es la empresa, en este sentido podríamos hablar de un enfoque de demanda. En segundo lugar, su principal elemento de interés es el impacto que una reducción de jornada puede generar sobre la creación de empleo y el nivel salarial. La tercera característica común de estos trabajos es la importancia que se le da a los costes laborales en la determinación del nivel de empleo y en el mayor o menor efecto de la las políticas de reducción de jornada. Por último, dado que se trata de una visión de demanda, se excluye el análisis del bienestar del individuo.

Los resultados de todos estos trabajos parecen ser coincidentes, señalando que una política destinada a reducir la jornada laboral, lejos de crear empleo, lo destruye. Entre los trabajos más destacados en este enfoque se encuentran el de Hart (1984), 
que analiza el papel de las horas extras en un contexto de reducción de la jornada laboral, distinguiendo el papel que desempeña un empleado antiguo y uno nuevo. En la misma línea se encuentra el trabajo de Calmorfos y Hoel (1988), con la diferencia de que este último incluye turnos de trabajo.

En este enfoque de demanda juega un papel clave el mecanismo mediante el cual se fijan los salarios y las horas trabajadas. Así Hoel (1984) y Calmorfs (1985) analizan la reducción de jornada en una economía donde hay un sindicato monopolístico, que determina el nivel de salarios. Los resultados de estos trabajos son ambiguos, tanto sobre el nivel de empleo como sobre el salario que perciben los trabajadores. A pesar de esto se desprende una idea clara: los trabajadores prefieren una jornada menor cuando actúan de manera colectiva, que cuando lo hacen de manera individual.

En un punto totalmente opuesto se encuentra el trabajo de Hoel y Vale (1985) donde es la empresa la que, de manera unilateral, decide el empleo y el salario. En este punto, la reducción es claramente negativa tanto sobre el empleo como sobre los salarios.

Posteriormente Booth y Schiantearelli (1987) plantean el análisis de la reducción de la jornada bajo distintas tipos de mecanismos de fijación salarial y de jornada. El resultado en todos los casos parece claro: la reducción de la jornada tiene resultados ambiguos a menos que esta política vaya acompañada de otra para aliviar los costes laborales o incrementar la productividad. Al margen de este resultado, uno de las ideas más sobresalientes que se desprende de este trabajo, es que cuando los sindicatos actúan como un monopolio, puede que no estén actuando de forma racional.

Una vez analizadas las consecuencias básicas de la reducción de la jornada, algunos autores han introducido en su análisis otros elementos adicionales. Así, Hoel (1986) señala que la reducción de la jornada laboral en una economía con dos sectores puede incrementar el desempleo de ambos. Calmorfs y Hoel $(1988,1989)$ por su parte analizan la relación entre empleo, tiempo de trabajo y tiempo operativo del capital. En general los resultados siguen siendo contundentes, la política de reducción de jornada no genera empleo.

Vemos por tanto que, desde esta perspectiva, llamémosla de demanda, las implicaciones son bastante negativas para la economía que implante esta política. Posteriores enfoques han seguido centrándose en el lado de la demanda, pero señalando ya en alguna medida el impacto de considerar aspectos de la oferta de trabajo. Entre los trabajos que analizan elementos parciales referidos a la oferta de trabajo destaca el de Leslie (1987) o el de Moselle (1996).

Al margen de esta literatura inicial, pero tomándola como base, han surgido en la pasada década dos líneas de investigación, que partiendo de una premisa común, el 
análisis de la reducción de la jornada laboral, ha planteado marcos de trabajo muy distintos. Por un lado la literatura centrada en el concepto de trabajo indivisible y por otro lado la literatura que gira en torno al concepto de emparejamientos.

La primera de estas corrientes se basa en el concepto de indivisibilidad del factor trabajo y la existencia de loterías. Esta línea de investigación se apoyaría en trabajos como los de Hansen (1985), Rogerson (1988) y Kydland (1995). En concreto, este último realiza un análisis exhaustivo del comportamiento cíclico del factor trabajo, señalando como elemento a considerar, a la hora de entender las fluctuaciones del mercado laboral, la producción en el hogar.

Un trabajo relacionado con el comportamiento cíclico del factor trabajo puede ser el de Dolado y Gómez (1995) que analiza los flujos de creación y destrucción de empleo en la economía española.

La aportación más importante sobre el impacto de la reducción de la jornada laboral, en un contexto de indivisibilidad del trabajo, y apoyada en los referentes mencionados, es el trabajo de Fitzgerald (1996,1998), el cual analiza el impacto de una reducción de la jornada laboral en un contexto de equilibrio general. De este trabajo se desprende que el establecimiento de un impuesto al trabajo, sobre los individuos con mayor salario, provoca que el empleo de todos los tipos de trabajadores se reduzca. No obstante, Fitzgerald (1996b) destaca como elemento esencial, que el conjunto de desempleados tiene caracter ísticas muy diferentes a los individuos que trabajan durante muchas horas, y que este desajuste entre empleados y desempleados puede limitar el efecto del empleo, provocando una reducción de la producción y de la productividad. Posteriormente Fitzgerald (1998) plantea un modelo de equilibrio general con agentes heterogéneos, que no es más que una extensión de lo ya planteado por Hornstein y Prescott (1993), señalando además la necesidad de incorporar elementos de la oferta de trabajo.

Un trabajo más reciente en este área es el de Osuna, Dolado y Rios-Rull (2000), que partiendo de un modelo con indivisibilidad del trabajo, introducen costes de desplazamiento al lugar de trabajo sometidos a congestión como mecanismo para permitir que se genere la sustitución horas-hombre que se observa en los datos. Los resultados de su trabajo son que la caída del número de horas de 40 a 35, incrementa el empleo, si bien la producción y la productividad se ven negativamente afectados.

La segunda de las corrientes señaladas anteriormente es la basada en la literatura de emparejamientos. El trabajo fundamental en esta literatura es el de Pissarides (1990, 2000). Pissarides plantea la negociación en el mercado de trabajo como una actividad económica en si misma. El elemento esencial en este contexto es el empa- 
rejamiento que se produce entre desempleado y vacante. A partir de este trabajo se ha desarrollado una extensa literatura donde resaltan trabajos como el de Mortensen y Pissarides (1994) que analizan los flujos de creación y destrucción de empleo en un modelo emparejamiento con una tasa de destrucción de empleo endógena, el de Merz (1995) y el de Mortensen y Pissarides (1999).

A raíz de estos artículos, y basándose en la existencia de una función de emparejamiento, se han planteado el análisis de los efectos de una reducción de la jornada laboral. El trabajo más relevante en este sentido es el de Marimon y Zilibotti (1999). La principal ventaja de este marco, y que hace distinto este trabajo del de Fizgerald (1998), es la posibilidad de analizar el proceso de creación de empleo como una actividad económica en si misma, al mismo tiempo que resulta plausible analizar el impacto directo que una política como la reducción de la jornada laboral tiene sobre el nivel de desempleo de la economía.

Estos autores desarrollan un modelo de equilibrio general con funciones de emparejamiento, tomando como referencia el caso en el que la empresa e individuo negocian libremente tanto el salario como las horas trabajadas. Además, las horas trabajadas y los trabajadores son perfectamente sustitutivos. En este trabajo se señala que el efecto neto de una reducción de la jornada dependerá de la tecnología de producción y de las preferencias del individuo. Asumiendo unas preferencias $\mathrm{GHH}^{3}$, los resultados sobre el empleo son ambiguos, si bien pequeñas reducciones en la jornada parecen incrementar el empleo. En general señalan la cautela como principal arma a la hora de aplicar estas políticas.

Siguiendo con el planteamiento de Marimon y Zilibotti el artículo de Rocheteau (2000) introduce el problema de azar moral en este contexto. Más recientemente Ortega (2000) destaca que la reducción de jornada puede incrementar el empleo y el bienestar si las expectativas de demanda baja de la empresa son suficientemente significativas y/o si el precio de las horas extras es pequeño.

Hasta ahora se ha hablado de los trabajos teóricos, los cuales han centrado su análisis en las repercusiones económicas de una reducción de jornada. Sin embargo no conviene ignorar que la reducción de jornada, como política de creación de empleo, ha sido implementada en mayor o menor medida en las dos últimas décadas por

\footnotetext{
${ }^{3}$ Greenwood, Hercowitz y Huffman (1988).
} 
diversas economías europeas. En este sentido, algunos trabajos se han centrado en la contrastación empírica de dicha política.

Uno de los primeros trabajos al respecto es el de Hunt $(1996,1999)$ que, usando datos de panel socio-económicos, analiza el impacto de la reducción de la jornada laboral en Alemania. Entre los principales resultados resalta el hecho de que una reducción de una hora estándar se ha traducido en una reducción real de 0,85 a 1 hora, lo cual ha puesto de manifiesto en parte el poco impacto de las horas extras. Además Hunt muestra como la presión sindical ha provocado en Alemania un incremento del salario por hora entre el $2 \%$ y el $3 \%$, lo que ha hecho que, pese a la reducción de jornada, las ganancias medias mensuales en determinadas industrias se haya mantenido constante.

Otro de los trabajos pioneros en la cuantificación de esta medida es el de Costa (1998) que, si bien no analiza directamente la reducción de la jornada como política económica, si obtiene una serie de resultados empíricos sobre la evaluación de las horas trabajadas en las últimas décadas. Costa señala que la jornada laboral ha caído en los últimos 100 años de 10 a 8 horas diarias. Por otro lado muestra que tanto los individuos más educados como los que tienen más renta, trabajan más horas. Resalta entre sus conclusiones el hecho de que el número de horas ofertadas por individuos con menores salarios puede haber caído en relación con aquellas que ostentan mayores salarios. De este hecho se deduce que los cambios observados en la oferta son muy cuantiosos en comparación con los cambios en la demanda.

Más recientemente Kapteyn et al. (2000) hacen una revisión de aquellas economías que han implantado la reducción de jornada como política para permitir el reparto de empleo. Entre otras, estos autores estudian el comportamiento de la economía francesa, que tal y como hemos indicado anteriormente ha sufrido continuas políticas de reducción de jornada. En la mayoría de los casos los resultados que se desprenden del trabajo no son demasiado optimistas. Tampoco en Bélgica las políticas de reducción han llegado a los objetivos pretendidos. En este sentido De Rongé y Molitor (1991) critican el comportamiento sindical de mantener las pretensiones antiguas a pesar de profundos cambios técnicos y organizativos producidos en el puesto de trabajo. Los resultados para Holanda parecen más alentadores según estos mismos autores, si bien en este país la reducción del desempleo no ha sido tan grande como se esperaba, si se ha creado empleo.

Si la literatura referente a la reducción de jornada ha sido amplia, no lo ha sido menos los trabajos que se han ocupado de estudiar las actividades que se realizan en el seno del hogar. Dentro de esta extensa literatura destaca el trabajo de Benhabib et al. (1990) donde se señala que la actividad del hogar representa un $28 \%$ del tiempo 
total disponible. Además estos autores ponen hincapié en el alto grado de sustitutibilidad entre el tiempo dedicado al mercado y el tiempo dedicado al hogar. En esta misma línea Nosal et al. (1992) analizan las implicaciones de introducir la producción en el hogar en los modelos de mercado de trabajo. Posteriormente Rios-Rull (1993) plantea un modelo que incorpora producción en el hogar y cualificación en la escuela. Por su parte Greenwood et al. $(1993,1995)$ hacen extensible el modelo analizando la producción en el hogar dentro de un contexto de ciclo vital.

\section{Modelo}

En esta sección se desarrolla el modelo básico que se utilizará como punto de partida, basado en Pissarides (1990, 2000).

\subsection{Emparejamientos}

El mercado laboral viene caracterizado por una función de emparejamiento estándar del tipo:

$$
m=m(u, v)
$$

donde $m$ denota el número de emparejamientos, $v$ el número de vacantes y $u$ la masa total de desempleados. Se trata de una función creciente en ambos argumentos, y con rendimientos constantes a escala.

Definiendo $\theta\left(=\frac{v}{u}\right)$ como la tirantez del mercado laboral, podemos derivar la tasa a la que una vacante de empleo encuentra un trabajador desempleado, $q(\theta)=m(u, v) / v$, y la tasa a la que un trabajador desempleado encuentra una vacante de empleo $\theta q(\theta)=m(u, v) / u$. Siguiendo el modelo de Pissarides la condición de estado estacionario viene dada por:

$$
d(1-u)=\theta^{1-\varepsilon} u
$$

donde $d$ es la tasa exógena a la que se destruyen empleos, para un valor de $0<\varepsilon<1$. 


\subsection{Empresas}

La tecnología de producción de la empresas representativa i viene dada por:

$$
Y_{i}=A G\left(N_{i}, h_{m i}\right)^{\lambda}
$$

donde $A$ es un parámetro exógeno que supondremos constante, $N_{i}$ es el número de individuos empleados en la empresa i, trabajando cada uno de ellos $h_{m i}$ unidades de tiempo.

En este modelo se asume, de manera implícita, que todas las empresas tienen la misma dotación de capital, y que este stock de capital es constante. Se define $G\left(N_{i}\right.$, $h_{m i}$ ) como el factor trabajo, y se asume que la función de producción tiene rendimientos decrecientes respecto a este factor $(0<\lambda<1)$.

Las empresas decidirán cuantos individuos contratar, $N_{i}$, así como el número de vacantes que abren en cada periodo, $V_{i}$. De esta forma, el flujo neto hacia la empresa $i$ viene dado por la ecuación dinámica:

$$
\dot{N}_{i}=q(\theta) V_{i}-d N_{i}
$$

Definiendo $\phi$ como el coste fijo de una vacante a cubrir ${ }^{4}$, el problema de una empresa representativa vendrá dado por:

$$
\begin{aligned}
& \underset{\left\{N_{i t} V_{i t}\right\}_{=0}^{\infty}}{\operatorname{Max}} \quad \Pi \text { Mit }=\int_{0}^{\infty} 0 e^{-r t}\left[Y_{i t}\left(N_{i t}, h_{m i t}\right)-w h_{m i t} N_{i t} ?-\phi V_{i t}\right] d t \\
& \text { s.a. } \\
& \begin{array}{c}
Y_{i t}=A G\left(N_{i t}, h_{m i t}\right)^{\lambda} \\
\dot{N}_{i t}=q(\theta) V_{i t}-d N_{i t} \\
N_{i 0}>0 \text { dado }
\end{array}
\end{aligned}
$$

donde $\Pi$ representa el beneficio. Además se asume que la empresa toma como dado el salario por hora $(w)$, para más tarde negociarlo con los trabajadores ${ }^{5}$. Resolviendo

${ }^{4}$ También pueden interpretarse como costes de contratación o costes de formación.

${ }^{5}$ En el artículo de Marimon y Zilliboti (1999) se asume que es el salario total el que las empresas toman como dado. 
el problema de la empresa se obtiene la condición de demanda de trabajo o condición de creación de empleo ${ }^{6}$ :

$$
p \frac{\partial G\left(N, h_{m}\right)}{\partial N}-w h_{m}-\frac{\phi}{q(\theta)}(r+d)=0
$$

donde:

$$
p=A \lambda G\left(N, h_{m}\right)^{\lambda-1}
$$

representa la productividad marginal del factor trabajo.

\subsection{Hogares}

Se comienza considerando el caso de un modelo sin producción en el hogar, donde las preferencias de los hogares vienen representadas por la siguiente función de utilidad:

$$
u\left(c_{m}, h_{m}\right)
$$

donde $c_{m}$ son los bienes de consumo (bienes producidos en el mercado) y $h_{m}$ es el tiempo destinado a trabajar en el mercado. La función de utilidad es continuamente diferenciable y cóncava, $u_{1}>0$ y $u_{2}<0$. Definiendo el ocio como $l=1-h_{m}$, y partiendo del supuesto de que los agentes no pueden prestar ni pedir prestado, de manera que el consumo de bienes de mercado será igual al salario total del individuo $\left(c_{m}=w h_{m}\right)$.

${ }^{6}$ Eliminamos los subíndices por simplicidad, al asumir un momento $t$ y una empresa representativa $i$. 


\subsection{Salarios}

Sean $U$ y $V$ respectivamente el valor presente descontado de un trabajador desempleado y de un puesto vacante. Y sea $W$ y $J$ los valores presentes descontados de un trabajador empleado y de una vacante ocupada, respectivamente. Asumiendo el salario por hora como dado, tendremos:

$$
\begin{gathered}
r U=u^{u}+\theta q(\theta)[W-U]^{7} \\
r V=-\phi+q(\theta)[J-V] \\
r W=u^{e}+d[U-W] \\
r J=p h_{m}-w h_{m}+d[V-J]
\end{gathered}
$$

donde $u^{e}$ y $u^{u}$ representan, respectivamente, las utilidades instantáneas de un individuo que se encuentra empleado y desempleado, y que vienen dadas por:

$$
\begin{aligned}
u^{e} & =u\left(w, h_{m}\right) \\
u^{u} & =u(z, 0)
\end{aligned}
$$

donde $z$ representa el subsidio de desempleo. Para simplificar, se asume que $u^{u}=u(z, 0)=0$.

Por último, en equilibrio la creación de vacantes viene determinada por la llamada condición de libre entrada $(V=0)$.

Se establece en primer lugar el caso de Laissez-Faire como referencia en nuestra economía, donde empresas y hogares negocian libremente tanto la jornada laboral como los salarios ${ }^{8}$, siendo estos últimos determinados siguiendo un proceso de negociación de Nash:

${ }^{7}$ En un mercado viable $W>U$.

${ }^{8}$ Posteriormente se analizará el caso en el que la jornada laboral venga establecida por ley, de manera que sólo se negocian los salarios. 


$$
\text { máx }(W-U)^{\beta}(J-V)^{1-\beta}
$$

donde $\beta$ representa el poder de negociación de los trabajadores, que supondremos exógeno y constante.

\section{Reducción de la Jornada con Perfecta sustitución Y SIN PRODUCCIÓN EN EL Hogar}

\subsection{Equilibrio de Laissez-Faire}

De forma similar al trabajo de Marimon y Zilibotti (1999), asumimos perfecta sustitución entre el número de horas trabajadas $h_{m}$ y el número de trabajadores $N$, de manera que la función de producción de la empresa representativa vendrá dada por:

$$
Y_{i}=A\left(N_{i} h_{m i}\right)^{\lambda}
$$

y la productividad marginal del trabajo por:

$$
p_{i}=A \lambda\left(N_{i} h_{m i}\right)^{\lambda-1}
$$

Además se parte de una función de emparejamiento de tipo Cobb-Douglas:

$$
m(u, v)=u^{\varepsilon} v^{1-\varepsilon}
$$

de manera que $q(\theta)=\theta^{-\varepsilon}$ denota la tasa a la que una vacante de empleo encuentra a un trabajdor desempleado y $\theta \mathrm{q}(\theta)=\theta^{1-\varepsilon}$ la tasa a la que un trabajador desempleado encuentra una vacante. Para que el número de emparejamientos sea estrictamente creciente en ambos argumentos asumiremos que $\varepsilon \in(0,1)$. El problema al que se enfrenta la empresa vendrá dado por:

$$
\begin{gathered}
\operatorname{Max}_{\left\{N_{i t} V_{i t}\right\}_{t=0}^{\infty}} \Pi_{i t}=\int_{0}^{\infty} e^{-r t}\left[A\left(N_{i t} h_{m i t}\right)^{\lambda}-w h_{m i t} N_{i t}-\phi V_{i}\right] d t \\
\text { s.a. } \dot{N}_{i t}=\theta^{-\varepsilon} V_{i}-d N_{i t} \\
N_{i 0}>0 \text { dado }
\end{gathered}
$$


resolviendo (19) obtendremos la condición de creación de empleo?:

$$
\theta^{-\varepsilon} h_{m}[p-w]=\phi(r+d)
$$

donde, como puede observarse, el valor esperado capitalizado de los costes de contratación de la empresa debe ser igual a la diferencia entre la productividad marginal menos el coste del factor trabajo ${ }^{10}$. Las preferencias vendrán caracterizadas por una función de utilidad CES:

$$
u\left(c_{m}, l_{m}\right)=\frac{\left[\mathrm{c}_{m}^{\alpha} l^{1-\alpha}\right]^{1-\sigma}}{1-\alpha}
$$

donde $\sigma>0$ y $\alpha \neq 1$. Por tanto tomando (21), la utilidad de un individuo empleado será:

$$
u^{e}=\frac{1}{1-\alpha}\left[\left(w h_{m}\right)^{\alpha}\left(1-h_{m}\right)^{1-\alpha}\right]^{1-\sigma}
$$

mientras que la utilidad de un individuo desempleado vendrá dada por:

$$
u^{u}=\frac{1}{1-\alpha} z^{\alpha(1-\sigma)}=0
$$

De acuerdo con las funciones de producción y emparejamientos especificas, las ecuaciones (9)-(12) pueden escribirse de la forma siguiente:

${ }^{9}$ Suponemos un momento $t$ y una empresa representativa $i$, eliminando por tanto los subíndices.

${ }^{10} \mathrm{Si}$ los costes de contratación fuesen nulos, $\phi=0$, tendríamos la condición clásica de que el salario es igual a la productividad marginal del trabajo $(p=w)$. 


$$
\begin{gathered}
r U=u^{u}+\theta 1^{-\varepsilon}[W-U] \\
r V=-\phi+\theta^{-\varepsilon}[J-V] \\
r W=u^{e}+d[U-W] \\
r J=[p-w] h_{m}+d[V-J]
\end{gathered}
$$

Resolviendo el programa de negociación de Nash dado por la expresión (15), llegamos a las siguientes condiciones de optimalidad ${ }^{11}$ :

$$
\begin{aligned}
& \frac{-\beta u^{e} h_{m}}{u^{e}-u^{u}}=\frac{(1-\beta)[p-w]}{[p-w] h_{m}+\phi \theta} \\
& \frac{-\beta u_{w}^{e}}{u^{e}-u^{u}}=\frac{(1-\beta) h_{m}}{[p-w] h_{m}+\phi \theta}
\end{aligned}
$$

donde $u_{w}^{e}$ representa la utilidad marginal del salario por hora y $u_{h m}^{e}$ la utilidad marginal del tiempo destinado a trabajar en el mercado. Resolviendo el sistema formado por (28) y (29), se obtienen los valores de salario por hora y del número de horas en nuestra economía de Laissez-Faire ${ }^{12}$ :

$$
\begin{aligned}
& h_{m}^{L F}=\frac{p^{L F}[(1-\beta)+\beta \alpha(1-\sigma)]-\phi \theta^{L F} \beta(1-\alpha)(1-\sigma)}{p^{L F}(1-\beta \sigma)} \\
& w^{L F}=\frac{p L F \alpha \beta\left[p^{L F}+\phi \theta^{L F}\right](1-\alpha)}{p^{L F}[(1-\beta)+\beta \alpha(1-\sigma)]-\phi \theta^{L F} \beta(1-\alpha)(1-\sigma)}
\end{aligned}
$$

\footnotetext{
${ }^{11}$ Ver Anexo 1.
}

12 Ver Anexo 2. 
Por último, teniendo en cuenta la condición de estado estacionario (2), conocida la función de emparejamiento (18) y sustituyendo (30) y (31) en (17) y (20), se obtienen las siguientes ecuaciones:

$$
\begin{gathered}
p \equiv p^{L F}\left(\theta^{L F}\right)=\frac{\phi \theta^{L F} \beta(1-\sigma)+\phi(d+r)\left(\theta^{L F}\right)^{\varepsilon}(1-\beta \sigma)}{1-\beta} \\
p^{L F}=A \lambda\left[\frac{\theta^{L F}\left\{p^{L F}[1-\beta)+\beta \alpha(1-\mathrm{s})\right]-\phi \theta^{L F} \beta(1-\alpha)(1-\sigma)}{(1-\beta \sigma) p^{L F}\left[q^{L F}+\left(q^{L F}\right)^{\varepsilon}\right]}\right]^{\lambda-1}
\end{gathered}
$$

con dos incógnitas, $\theta^{L F}$ y $p^{L F}$. Como podemos observar la ecuación (32) recoge la productividad marginal en función exclusivamente de los parámetros y del valor de $\theta L F$. Por último, sustituyendo (32) en (33) se deriva la siguiente expresión:

$$
\begin{gathered}
\Gamma^{L F}\left(\theta^{L F}\right) \equiv \frac{\phi \theta^{L F} \beta(1-\sigma)+\phi(d+r)\left(\theta^{L F}\right)^{\varepsilon}(1-\beta \sigma)}{1-\beta} \\
-A \lambda\left[\frac{\theta^{L F}\left[(d+r)(1-\beta)\left(\theta^{L F}\right)^{\varepsilon}+\alpha \beta(1-\sigma)\left[\theta^{L F}+(d+r)\left(\theta^{L F}\right)^{\varepsilon}\right]\right]}{\left[\theta^{L F}+d\left(\theta^{L F}\right)^{\varepsilon}\right]\left[\beta \theta^{L F}(1-\sigma)+(d+r)\left(\theta^{L F}\right)^{\varepsilon}(1-\beta \sigma)\right]}\right]=0
\end{gathered}
$$

que define la solución para $\theta^{L F}$.

Sustituyendo $\theta^{L F}$ y el valor de la productividad marginal dado por (32) en las expresiones (30) y (31), se tiene:

$$
\begin{gathered}
h_{m}^{L F}(\theta L F)=\frac{(d+r)(1-\beta)\left(\theta^{L F}\right)^{\varepsilon}+\alpha \beta\left[\theta^{L F}+(d+r)\left(\theta^{L F}\right)^{\varepsilon}\right](1-\sigma)}{\beta \theta^{L F}(1-\sigma)+(d+r)\left(\theta^{L F}\right)^{\varepsilon}(1-\beta \sigma)} \\
w^{L F}(\theta L F)=\frac{\phi \alpha \beta\left[\theta^{L F}+(d+r)\left(\theta^{L F}\right)^{\varepsilon}\right](1-\sigma)\left[\mathrm{b} \theta^{L F}(1-\sigma)+(d+r)\left(\theta^{L F}\right)^{\varepsilon}(1-\beta \sigma)\right]}{(1-\beta)\left[(d+r)(1-\beta)\left(\theta^{L F}\right)^{\varepsilon}+\alpha \beta(1-\sigma)\left[\theta^{L F}+(d+r)\left(\theta^{L F}\right)^{\varepsilon}\right]\right]}
\end{gathered}
$$


Definición 1: El equilibrio de Laissez-Faire en una economía sin producción en el hogar viene caracterizado por el vector $\left(\theta^{L F}, p^{L F}, w^{L F}, h_{m}^{L F}\right)$ que satisface las condiciones (30)-(32) y (34).

Tal y como se ha indicado al comienzo, uno de los elementos relevantes del análisis es medir el efecto que tienen las políticas de reducción de jornada sobre el bienestar de los individuos. Para ello se establece una medida de bienestar en función del valor presente de estar desempleado, ecuación (24), y empleado, ecuación (26).

Definición 2: El nivel de bienestar de los individuos de una economía de Laissez-Faire, BLF , viene dado por la siguiente expresión:

$$
B^{L F}=(1-u)[r W]+u[r U]
$$

A partir de la Definición 2, tomando (24) y (26), y conocidos los valores (22) y (23) en equilibrio, (37) puede escribirse de la forma siguiente ${ }^{13}$ :

$$
B^{L F}=(1-u) r u^{e}\left(h_{m}^{L F}, w^{L F}\right)
$$

\subsection{Equilibrio con Jornada Laboral Restringida}

Supongamos ahora que el tamaño de la jornada laboral viene fijado por ley. En este caso el programa de negociación salarial se reduce a:

$$
\operatorname{máx}_{\{w\}}(W-U)^{\beta}(J-V)^{1-\beta}
$$

que conduce a la siguiente expresión para el salario ${ }^{14}$ :

\footnotetext{
${ }^{13}$ Ver Anexo 3.
}

${ }^{14}$ Ver Anexo 4. 


$$
w_{\text {máx }}^{R}=p^{R}+\frac{(1-\sigma) \alpha \beta\left[p+\frac{\phi \theta^{R}}{h_{m}^{R}}\right]}{(1-\beta)+\alpha \beta(1-\sigma)}
$$

A partir de (40) podemos ver, tal y como señalan Marimon y Zilliboti (1999), que si los hogares tuvieran todo el poder de la negociación es decir, si $\beta=1$, el salario alcanzaría su nivel máximo:

$$
w_{\text {máx }}^{R}=p^{R}+\frac{\phi \theta^{R}}{h_{m}^{R}}
$$

que como podemos observar, es igual a la suma de la productividad marginal y una proporción de los costes asociados a la contratación. Análogamente al apartado de Laissez-Faire, teniendo en cuenta la condición de estado estacionario (2) y la función de emparejamiento (18), y sustituyendo (40) en (17) y (20), se derivan las siguientes ecuaciones:

$$
\begin{gathered}
p=p^{R}\left(\theta^{R}\right)=A \lambda\left[\frac{\theta^{R}}{d\left(\theta^{R}\right)^{\varepsilon}+\theta^{R}} h_{m}^{R}\right]^{\lambda-1} \\
p^{R}=\frac{[(1-\beta)+\alpha \beta(1-\sigma)] \phi(d+r)}{\left(\theta^{R}\right)^{-\varepsilon} h_{m}^{R}(1-\beta)}+\frac{(1-\sigma) \alpha \beta \phi \theta^{R}}{h_{m}^{R}(1-\beta)}
\end{gathered}
$$

Igualando (42) y (43) llegamos a la siguiente expresión:

$$
\Phi^{R}\left(\theta^{R}\right) \equiv \frac{A h_{m}^{R}(1-\beta) \lambda\left[\frac{\theta^{R}}{d\left(\theta^{R}\right)^{\varepsilon}+\theta^{R}}\right]^{\lambda-1}-\phi \alpha \beta \theta^{R}(1-\sigma)}{(1-\beta)+\alpha \beta(1-\sigma)}-\phi(d+r)\left(\theta^{R}\right)=0
$$

que define la solución para $\theta^{R}$ en un equilibrio con jornada laboral restringida.

Por otro lado, podemos calcular el salario por hora en función de $\theta^{R}$, sustituyendo (42) en (40):

$$
w^{R}=\frac{(1-\sigma) \alpha \beta\left[A \lambda\left[\frac{\theta^{R}}{d\left(\theta^{R}\right)^{\varepsilon}+\theta^{R}} h_{m}^{R}\right]^{\lambda-1}+\frac{\phi \theta^{R}}{h_{m}^{R}}\right]}{(1-\beta)+\alpha \beta(1-\sigma)}
$$


Definición 3: En un modelo sin producción en el hogar y con jornada laboral fija, hRm, el Equilibrio Restringido viene caracterizado por el vector $\left(\theta^{R}, p^{R}, w^{R}\right)$, que satisface las condiciones (40), (42) y (44).

Definición 4: El nivel de bienestar de una economía sin producción en el hogar y jornada laboral restringida viene dado por $B^{R}=r(1-u) u^{e}\left(h_{m}^{R}, w^{R}\right)$.

Proposición 1: En un modelo sin producción en el hogar y con jornada laboral fija, una política de reducción de jornada, aumenta la tasa de desempleo en estado estacionario.

Demostración: Ver anexo 5.

Según este resultado una política de reducción de jornada, como mecanismo de creación de empleo, no resulta efectiva, dado que no sólo no consigue crear empleo, sino que lo destruye. Este resultado se debe a que la reducción de jornada disminuye la probabilidad de que un desempleado encuentre una vacante $\left(\theta^{1-\varepsilon}\right)$, incrementando de esta forma el nivel de desempleo de equilibrio.

Además, a partir de la proposición anterior, es fácil demostrar que una política de reducción de jornada disminuye la productividad del factor trabajo ${ }^{15}$.

El efecto de la reducción de jornada sobre el salario por hora resulta ambiguo ${ }^{16}$. Por un lado, de la ecuación (42) tenemos que la productividad marginal del factor trabajo se incrementa al reducirse la jornada, lo que provoca un incremento del salario por hora en equilibrio. Pero por otro lado, una reducción de jornada conduce a una disminución del salario por hora vía reducción de $\theta^{R}$. Por tanto, en principio, no es posible saber cuál será el efecto de una reducción de jornada sobre los salarios. No obstante, podemos demostrar que hay un umbral por debajo del cual la reducción de jornada lleva a un incremento del nivel salarial por hora de equilibrio.

Proposición 2: Sea $\theta^{*}$ el umbral superior de $\theta$ que satisface la ecuación: $\left[\frac{\mathrm{A} \lambda(1-\lambda) d(1-\varepsilon)}{\phi}\right]^{\lambda} h_{m}=\left(\theta^{*}\right)^{\frac{2-\varepsilon}{\gamma}}\left[1+d\left(\theta^{*}\right)^{(\varepsilon-1)}\right]$, y sea $\theta^{R}$ el valor de equilibrio de $\theta$.

${ }^{15}$ Ver Anexo 6.

${ }^{16}$ Ver Anexo 7. 
Si $\theta^{R} \leq \theta^{*}$, la reducción de la jornada laboral provocará un aumento del salario por hora de equilibrio.

\section{Demostración: Ver Anexo 7.}

El resultado de la Proposición 2 está en línea con algunos trabajos empíricos. Por ejemplo, para la economía alemana Hunt (1999) muestra que la reducción de jornada a 35 horas incrementa el salario por hora.

Por último, determinando el efecto de la reducción de jornada sobre el nivel de Bienestar de los individuos de la economía podemos ver que el resultado no es concluyente ${ }^{17}$.

Los resultados obtenidos están en consonancia con los expuestos por Marimon y Zilibotti (1999). Si bien este análisis lleva consigo dos claras diferencias en cuanto al trabajo de dichos autores. La primera es el hecho, como ya se ha mencionado, de restringir la elasticidad de sustitución entre ocio y trabajo igual a la unidad, necesario para estar acorde con la evidencia empírica existente. La segunda radica en ampliar los grados de libertad tanto de la elasticidad de sustitución intertemporal $(\sigma)$, como del peso del consumo en la utilidad del individuo $(\alpha)$. En el presente trabajo hemos asumido un rango más amplio para el parámetro $\sigma$, a diferencia de Marimon y Zilibotti que asumen igual a 0 . Por otro lado, ellos fijan directamente la participación del consumo en $1 / 2$, mientras que en este trabajo se ha relajado este supuesto, para calibrar más convenientemente el consumo dentro de la función de utilidad. Dada la equivalencia de los modelos es fácil de comprobar, y de hecho ya se ha hecho implícitamente, el cumplimiento de la Proposición 6 del trabajo de Marimon y Zilibotti (1999), que establece para el caso donde $\xi=0$ (caso equivalente al de esta sección), que el efecto de la reducción será inequívocamente de destrucción de empleo.

El objetivo principal de la siguientes secciones es, por un lado analizar el efecto de una reducción de jornada en un contexto con producción en el hogar, y posteriormente comparar los resultados con los obtenidos en esta sección.

${ }^{17}$ Ver Anexo 8. 


\section{Reducción de la Jornada Laboral con Producción en el Hogar y Perfecta Sustitución Horas-Hombre}

En esta sección se analiza un modelo donde las horas y los individuos siguen siendo perfectamente sustitutivos, pero donde los individuos pueden destinar su dotación de tiempo, no sólo a trabajar en el mercado y al ocio, sino a realizar actividades en el seno del hogar. Ahora los trabajadores influyen en el nivel de desempleo, no sólo a través del salario, sino también a través de la productividad de los agentes en actividades del hogar. Se asume, además, que dicha actividad será idéntica para todos los individuos y que los hogares no pueden prestar ni pedir prestado. La nueva función de utilidad vendrá dada por $u\left(c_{m h}, c_{n}, h_{m h}, h_{n}\right)$, donde $c_{m h}$ representa el consumo de bienes producidos en el mercado, $c_{n}$ el consumo de bienes producidos en el hogar, hmh la proporción de tiempo destinado a trabajar en el mercado laboral y $h_{n}$ el tiempo destinado a trabajar en el hogar. El individuo dispone de una unidad de tiempo en cada período, de manera que podemos definir el ocio como: $l=1-h_{m h}-h_{n}$. Además se asume que $\mathrm{u}(\cdot)$ es continuamente diferenciable y cóncava, $u_{1}>0, u_{2}>0, u_{3}<0 \mathrm{y}$ $u_{4}<0$.

La función de producción en el hogar viene dada por:

$$
c_{n}=g\left(h_{n}\right)
$$

donde $g$ define la tecnología de producción en el hogar, la cual depende exclusivamente del tiempo destinado a producir en el hogar. Dicha función es creciente y cóncava. Un elemento importante en este modelo es la existencia de una asimetría clara entre unos y otros bienes ya que los bienes de producción en el hogar, a diferencia de los bienes de mercado, sólo se pueden destinar a consumir en el hogar.

Podemos definir la función de utilidad de forma reducida como sigue:

$$
v\left(c_{m h}, h_{m h}\right) \equiv \operatorname{máx}_{h n} u\left(c_{m h}, \mathrm{~g}\left(h_{n}\right), h_{m h}, h_{n}\right)
$$

Es fácil demostrar que esta función de utilidad es continua y creciente en $c_{m h} \mathrm{y}$ $h_{m h}$, así como cóncava en ambos argumentos.

De acuerdo con lo planteado por Benhabib et al. (1990) (Teorema 1, página 7), se puede establecer la siguiente proposición.

Proposición 3: Supongamos que $u(\cdot)$ y $g(\cdot)$ son funciones estrictamente cónca- 
vas. Definiendo $h_{n}=h\left(c_{m h}, h_{m h}\right)$ como el tiempo de trabajo en el hogar, podemos decir que hn será la única solución al problema de maximización definido por (47), sujeto a $h_{n} \in\left[0,1-h_{m h}\right]$, y la función de consumo en el hogar será $c_{n}=c\left(c_{m h}, h_{m h}\right)=g \circ h$ $\left(c_{m h}, h_{m h}\right)$. Al introducir la producción en el hogar, las expresiones (9) y (11) quedarían de la forma siguiente:

$$
\begin{gathered}
r U=v(z, 0)+\theta \mathrm{q}(\theta)[W-U] \\
r W=v\left(c_{m h}, h_{m h}\right)+d[U-W]
\end{gathered}
$$

donde $v\left(c_{m h}, h_{m h}\right)$ y $v(z, 0)$ definen respectivamente el valor de la utilidad en forma reducida de un individuo empleado y desempleado cuando hay producción en el hogar. Además podemos simplificar el modelo asumiendo que $v(z, 0)=0$.

Bajo estos nuevos supuestos se procede en primer lugar a plantear el equilibrio de Laissez Faire, y en segundo lugar el equilibrio con jornada laboral fija.

\subsection{Equilibrio de Laissez Faire}

Dado el supuesto de perfecta sustitución horas-hombre, la condición de creación de empleo sigue siendo la correspondiente a la ecuación (20). Además, se supone una función de utilidad tipo CES:

$$
U(C, l)=\frac{\left[C \alpha l^{1-\alpha}\right]^{1-\sigma}}{1-\sigma}
$$

donde $C$ es el nivel de consumo total del hogar, $l$ el ocio y $\alpha$ la participación del consumo dentro de la utilidad del individuo. De acuerdo con los modelos de producción en el hogar, asumimos que el consumo total $\mathrm{C}$ viene definido por:

$$
C=\left[a c_{m h}^{e}+b c_{n}^{e}\right]^{\frac{1}{e}}
$$

La elasticidad de sustitución entre $c_{m h}$ y $c_{n}$ es $1 / 1-e$, que supondremos igual a la unidad (es decir $e=0$ ). Por tanto $C=\mathrm{c}_{m h}^{a} c_{n}^{b}$, donde $a$ y $b$ representan, respectivamente, los pesos de la variación de $c_{m h}$ y $c_{n}$ sobre la variación total del consumo $(0<a, b<1$, $a+b=1)$. Supongamos que la tecnología de producción en el hogar viene dada por: 


$$
g\left(h_{n}\right)=h_{n}^{\eta}
$$

donde $0<\eta<1, \frac{\partial g\left(h_{n}\right)}{\partial h_{m}}>0, \frac{\partial^{2} g\left(h_{n}\right)}{\partial\left(h_{n}\right)^{2}}<0$. Tenemos, por tanto, rendimientos decrecientes en la producción en el hogar. Los hogares deben decidir primero el tiempo que van a dedicar a actividades del hogar. El problema de los hogares vendrá dado por:

$$
\begin{aligned}
\operatorname{máx}_{h_{n}} U(\mathrm{C}, l) & =\frac{\left[\mathrm{C}^{\alpha} l^{1-\alpha}\right]^{1-\alpha}}{1-\alpha} \\
\text { s.a. } \quad \quad & =c_{m h}^{a} c_{n}^{b} \\
c_{n} & =g\left(h_{n}\right)=h_{n}^{\eta} \\
l & =1-h_{m h}-h_{n}
\end{aligned}
$$

Resolviendo (53) obtendremos la función de oferta de trabajo en el hogar:

$$
h_{n}=h\left(h_{m h}\right)=\frac{\alpha b \eta\left(1-h_{m h}\right)}{(1-\alpha)+\alpha b \eta}
$$

Examinando la ecuación (54), puede observarse que el tiempo destinado a actividades del hogar depende negativamente del tiempo destinado al trabajo. Además, puede verse que si $h_{m h}$ viniese fijado por ley, el efecto del parámetro $\eta$ es claramente positivo, es decir, cuanto más alta sea la participación del tiempo en actividades del hogar en la producción de bienes del hogar, mayor será el nivel óptimo de tiempo que dediquen los hogares a esta actividad. Igualmente, cuanto mayor sea el peso del consumo de bienes del hogar sobre el consumo total, b, más alto será el tiempo que los individuos destinen a actividades del hogar.

Sustituyendo (54) en (47) obtenemos la siguiente expresión para la función de utilidad en forma reducida:

$$
v\left(c_{m h}, h_{m h}\right)=\frac{1}{1-\sigma}\left[c_{m h}^{\chi}\left(1-h_{m h}\right)^{\gamma}\right]^{1-\sigma}
$$

donde $\chi=\mathrm{a} \alpha \mathrm{y}=\alpha b \eta+(1-\alpha)$, la cual se obtiene previa transformación lineal. A partir de (55) podemos derivar la utilidad instantánea de un individuo empleado $\left(v^{e}\right)$ y desempleado $\left(v^{u}\right)$, teniendo en cuenta que la renta salarial total $w_{h} h_{m h}$ se destina íntegramente al consumo de bienes de mercado: 


$$
\begin{gathered}
v^{e}=v\left(w_{h}, h_{m h}\right)=\frac{1}{1-\sigma}\left[\left(w_{h} h_{m h}\right)^{\chi}\left(1-h_{m h}\right)\right]^{1-\sigma} \\
v^{u}=v(z, 0)=\frac{1}{1-\sigma}\left[z^{\chi}\right]^{1-\sigma}
\end{gathered}
$$

donde asumiremos que $v^{u}=0$.

Una vez caracterizado el comportamiento de los hogares, se plantea de nuevo el problema de negociación, donde empresa y hogares deciden mediante un proceso de negociación de Nash el salario por hora y el tiempo que los individuos destinan a actividades de mercado. Resolviendo (15) para los nuevos supuestos obtenemos las siguientes condiciones de optimalidad ${ }^{18}$ :

$$
\begin{gathered}
h_{m h}^{L F}: \frac{-\beta v_{h_{m h}}^{e}}{v^{e}-v^{u}}=\frac{(1-\beta)\left[p_{h}-w_{h}\right]}{\left[p_{h}-w_{h}\right] h_{m h}+\phi \theta_{h}} \\
w_{h}^{L F}: \frac{\beta v_{h_{m h}}^{e}}{v^{e}-v^{u}}=\frac{(1-\beta) h_{m h}}{\left[p_{h}-w_{h}\right] h_{m h}+\phi \theta_{h}}
\end{gathered}
$$

Sustituyendo los valores de $v_{h_{m h}}^{e}$ y $v_{w}^{e}$ en (58) y $(59)^{19}$, obtenemos:

$$
\begin{gathered}
h_{m h}^{L F}=\frac{\beta \chi(1-\sigma)+(1-\beta)-\beta \gamma(1-\sigma) \frac{\phi \theta^{L F}}{p_{h}^{L F}}}{\beta \chi(1-\sigma)+(1-\beta)+\beta \gamma(1-\sigma)} \\
h_{m h}^{L F}=\frac{\beta \chi(1-\sigma)\left[\phi \theta^{L F}+p_{h}^{L F}\right]}{\beta \chi(1-\sigma)+(1-\beta)-\beta \gamma(1-\sigma) \frac{\phi \theta^{L F}}{p_{h}^{L F}}}
\end{gathered}
$$

\footnotetext{
${ }^{18}$ Ver Anexo 9.

${ }^{19}$ Ver Anexo 10.
} 
Siguiendo el mismo procedimiento de las secciones anteriores, utilizando (2), (17), (20), (60) y (61) llegamos a las siguientes expresiones:

$$
\begin{gathered}
p_{h}^{L F}\left(\theta_{h}^{L F}\right)=\frac{\phi \theta_{h}^{L F} \beta(1-\sigma)(\gamma+\chi)+\phi(d+r)\left(\theta_{h}^{L F}\right)^{\varepsilon}[1-\beta+\beta(1-\sigma)(\gamma+\chi)]}{1-\beta} \\
\mu\left(\theta_{h}^{L F}\right)=\frac{\phi \theta_{h}^{L F} \beta(1-\sigma)(\gamma+\chi)+\phi\left(\theta_{h}^{L F}\right)^{\varepsilon}(d+r)[1-\beta+\beta(1-\sigma)(\gamma+\chi)]}{1-\beta}-A \lambda \mathrm{D}^{(\lambda-1)}=0
\end{gathered}
$$

donde $\theta_{h}^{L F}$ es el valor de la tirantez en estado estacionario, $p_{h}^{L F}$ el valor de la productividad y $\Delta$ es una función de los parámetros del modelo ${ }^{20}$.

A partir del valor de $p_{h}^{L F}\left(\theta_{h}^{L F}\right)$ podemos obtener los valores para el salario hora y la jornada laboral de Laissez-Faire en función únicamente de $\theta_{h}^{L F}$, sustituyendo (62) en (60) y (61):

$$
\begin{gathered}
h_{m h}^{L F}=\frac{\beta \theta_{h}^{L F} \chi(1-\sigma)+(d+r)\left(\theta_{h}^{L F}\right)^{\varepsilon}[(1-\beta)+\beta \chi(1-\sigma)]}{\beta \theta_{h}^{L F}(\chi+\gamma)(1-\sigma)+(d+r)\left(\theta_{h}^{L F}\right)^{\varepsilon}[(1-\beta)+\beta(\chi+\gamma)(1-\sigma)]} \\
w_{h}^{L F}=\frac{\phi \beta(1-\sigma)}{\left\{\beta \theta_{h}^{L F}(1-\sigma) \chi+(d+r)\left(\theta_{h}^{L F}\right)^{\varepsilon}[(1-\beta)+\beta \chi(1-\sigma)]\right\}} \cdot \\
\left\{\frac{\beta\left(\theta_{h}^{L F}\right)^{2}(1-\sigma)(\chi+\gamma)+(d+r)\left(\theta_{h}^{L F}\right)^{2 \varepsilon}[(1-\beta)+\beta \chi(1-\sigma)]}{(1-\beta)}+\right. \\
\left.\frac{\left\{(d+r)\left(\theta_{h}^{L F}\right)^{1+\varepsilon}[(1-\beta)+2 \beta \gamma(1-\sigma)+2 \chi(1-\sigma)]\right\}}{(1-\beta)}\right\}
\end{gathered}
$$

Definición 5: El equilibrio de Laissez-Faire en un modelo con producción en el hogar viene caracterizado por el vector $\left(\theta_{h}^{L F}, p_{h}^{L F}, w_{h}^{L F}, h_{m h}^{L F}, h_{n}^{L F}\right)$ que satisface las condiciones (54), (60)-(63).

${ }^{20}$ Ver Anexo 11. 
Definición 6: El nivel de bienestar de los individuos de una economía de Laissez-Faire con producción en el hogar, BLF h, viene dado por la siguiente expresión:

$$
B_{h}^{L F}=(1-u) r v^{e}\left(w_{h}^{L F}, h_{m}^{L F}\right)
$$

En la siguiente sección comparamos estos resultados con los obtenidos para una economía sin producción en el hogar.

\subsection{Jornada Laboral Restringida}

En esta sección nos centramos en el caso en que la jornada laboral, hR mh, viene fijada por ley, de manera que las empresas y los hogares sólo deciden el salario por hora. Resolviendo (39) se llega a la siguiente condición de optimalidad ${ }^{21}$ :

$$
w_{h}^{R}=\frac{\beta \chi(1-\sigma)\left[p+\frac{\phi \theta_{h}^{R}}{h_{m h}^{R}}\right]}{(1-\beta)+\beta \chi(1-\sigma)}
$$

Teniendo en cuenta la condición de estado estacionario (2), y la función de emparejamiento (18) y sustituyendo (67) en (17) y (20):

$$
\begin{gathered}
p=p_{h}^{R}\left(\theta_{h}^{R}\right) A \lambda\left[\frac{\theta_{h}^{R}}{d\left(\theta_{h}^{R}\right)^{\varepsilon}+\theta_{h}^{R}} h_{m h}^{R}\right]^{\lambda-1} \\
p=p_{h}^{R}\left(\theta_{h}^{R}\right)=\frac{[(1-\beta)+\beta \chi(1-\sigma)] \phi(d+r)}{d\left(\theta_{h}^{R}\right)^{\varepsilon}+h_{m h}^{R}(1-\beta)}+\frac{(1-\sigma) \chi \beta \phi \theta_{h}^{R}}{h_{m h}^{R}(1-\beta)}
\end{gathered}
$$

Igualando (68) y (69) se obtiene:

${ }^{21}$ Ver Anexo 12. 


$$
\frac{A h_{m h}^{R}(1-\beta) \lambda\left(\frac{h_{m h}^{R} e_{h}^{R}}{\theta_{h}^{R}+d\left(\theta_{h}^{R}\right)^{\varepsilon}}\right)^{\lambda-1}-\phi \theta_{h}^{R} \beta \chi(1-\sigma)}{(1-\beta)+\beta \chi(1-\sigma)} \phi\left(\theta_{h}^{R}\right)^{\varepsilon}(d+r)
$$

que define la solución para $\theta_{h}^{R}$ en un equilibrio con jornada laboral restringida. Por último, sustituyendo (68) en (67) se obtiene el salario por hora en función de $\theta_{h}^{R}$ :

$$
w_{h}^{R}\left(\theta_{h}^{R}, h_{m h}^{R}\right)=\frac{\beta \chi(1-\sigma)\left[\phi \theta_{h}^{R}+A h_{m h}^{R} \lambda\left(\frac{h_{m h}^{R} \theta_{h}^{R}}{d\left(\theta_{h}^{R}\right)^{\varepsilon}+\theta_{h}^{R}}\right)^{\lambda-1}\right]}{h_{m h}^{R}[(1-\beta)+\beta \chi(1-\sigma)]}
$$

Definición 7: En un modelo con producción en el hogar y con jornada laboral fija, $h_{m h}^{R}$, el equilibrio restringido viene caracterizado por el vector $\left(\theta_{h}^{R}, p_{h}^{R}, w_{h}^{R}, h_{n}^{R}\right)$ que satisface las condiciones (54), (67), (68) y (70).

Definición 8: El nivel de bienestar de una economía con producción en el hogar y jornada laboral restringida viene dado por: $B_{h}^{R}=(1-u) r v^{e}\left(w_{h}^{R}, h_{m h}^{R}\right)$.

Al igual que en el modelo sin producción en el hogar, podemos analizar que implicaciones tendría una política de reducción de jornada. Se empieza estudiando el efecto sobre el tiempo que los individuos destinan a actividades del hogar, y por tanto también al ocio. A partir de la condición (54) tenemos:

$$
\begin{aligned}
\frac{d h_{n}^{R}}{d h_{m h}^{R}} & =\frac{\alpha b \eta}{(1-\alpha)+\alpha b \eta}<0 \\
\frac{d l_{h}}{d h_{m h}^{R}} & =\frac{(1-\alpha)}{(1-\alpha)+\alpha b \eta}<0
\end{aligned}
$$

Como puede observarse, la reducción de la jornada laboral lleva tanto a un incremento del tiempo de ocio como del tiempo que los individuos destinan a actividades del hogar. No obstante, no podemos saber a priori cuál de las dos actividades se verá más afectada por la reducción de la jornada, si bien podemos desterrar la idea desarrollada por algunos autores de considerar que la reducción de jornada llevará acarreado exclusivamente un incremento del tiempo destinado al ocio. El hecho de no 
considerar las actividades del hogar conduciría a sobredimensionar el efecto de la reducción de jornada sobre el tiempo dedicado al ocio.

Proposición 4: $\mathrm{Si}(1-\alpha)>\alpha b \eta$, el efecto de la reducción de jornada tendrá una mayor repercusión en el ocio de los individuos, mientras que si $(1-\alpha)<\alpha b \eta$, serán las actividades del hogar las beneficiadas por la reducción de jornada.

Demostración: Ver anexo 13.

Esta proposición sugiere que si la participación del ocio dentro de la función de utilidad es suficientemente alta, la reducción de jornada repercutirá más en esta actividad. Si por el contrario los agentes ponderan poco el ocio, la reducción de jornada llevará a un incremento mayor de las actividades del hogar que de las actividades de ocio. En este sentido, es probable que los hogares con hijos ponderen menos el tiempo destinado a ocio dentro de su función de utilidad y más el consumo de bienes, ya sea obtenidos en el mercado, o producidos en el hogar. En este contexto, una reducción de jornada implicaría un mayor efecto en el tiempo destinado a las actividades del hogar que en el tiempo destinado al ocio. Por otro lado, una reducción de jornada incrementará el consumo de bienes producidos en el hogar, lo que podría conducir a la existencia de una economía sumergida:

$$
\frac{d c_{n}}{d h_{m h}^{R}}=\frac{d g\left(h_{n}^{R}\right)}{d h_{m h}^{R}}=\eta h_{n}^{n-1}\left[\frac{d h_{n}^{R}}{d h_{m h}^{R}}\right]<0
$$

Una vez analizado el impacto de la política de reducción sobre las variables más especificas del modelo con hogar, no conviene olvidar el estudio de esta medida en las variables relacionadas con el empleo y el salario.

Proposición 5: En un modelo con producción en el hogar y jornada laboral fija, una política de reducción de jornada, aumenta la tasa de desempleo en estado estacionario.

Demostración: Ver anexo 14.

A partir de esta proposición, es fácil de comprobar que una reducción de jornada provoca un descenso en el nivel de productividad. Además, tal y como se obtenía en el modelo sin producción en el hogar, el efecto sobre el salario de equilibrio resulta ambiguo. No obstante, al igual que en la sección anterior, si fijamos un valor de $\theta_{h}^{R}$ 
por debajo de $\theta^{*}$ (ver Proposición 2), la reducción de jornada laboral provocará que el salario por hora de equilibrio se incremente ${ }^{22}$.

Un aspecto interesante que no conviene pasar por alto, es el papel del peso que tiene el consumo de bienes de mercado $(a)$. Cuanto mayor es a, menor es el nivel de _ R h en estado estacionario para una jornada dada, disminuyendo así la probabilidad de encontrar una vacante para un individuo desempleado ${ }^{23}$. El único parámetro que identifica la producción en el hogar en los valores de equilibrio de $\theta_{h}^{R}$ y $w_{h}^{R}$ es $a$. Si a tiene un valor muy bajo, los hogares dan poco peso a los bienes de mercado y mucho a los bienes del hogar. Si la participación del consumo del hogar sobre el consumo total aumenta (a se reduce), el impacto negativo de la reducción de jornada sobre el empleo es mucho mayor.

\section{Comparación de Resultados}

En esta sección se analiza cuáles son las diferencias claves que supone la introducción del hogar en un contexto de equilibrio general donde hay una perfecta sustitución horas-hombre. La introducción de las actividades del hogar en un modelo de estas características nos hace darnos cuenta de la sobreestimación que se hace del ocio. Además, resulta obvio que en una economía donde los agentes producen bienes en el hogar, el peso de los bienes de consumo de mercado dentro de la función de utilidad es menor $(\chi=a \alpha<\alpha)$.

El siguiente paso es analizar la repercusión que tiene la introducción del sector del hogar sobre las principales variables de interés.

${ }^{22}$ Ver Anexo 5 y Anexo 6.

${ }^{23}$ Ver Anexo 17. 


\subsection{Equilibrio de Laissez-Faire}

Proposición 6: $\operatorname{Si} a+b \eta-\alpha \geq 0$ y suponiendo $\theta=\theta^{L F}=\theta_{h}^{L F}$, entonces $h_{m}^{L F}>h_{m h}^{L F}$. Demostración: Ver anexo 18.

Esta proposición nos indica que si el peso de los bienes de consumo y de ocio es suficientemente alto en la función de utilidad en forma reducida, los individuos elegirán siempre una jornada laboral más pequeña en un modelo con producción en el hogar. Este resultado esta en línea con la idea de que si los individuos saben que parte de su tiempo que no destinan a trabajar en el mercado es productivo fuera de él, querrán disponer de más tiempo fuera de su lugar de trabajo.

Proposición 7: Suponiendo un valor de $\theta$ dado, la productividad marginal del factor trabajo de Laissez-Faire en una economía sin producción en el hogar, $p^{L F}$, es estrictamente mayor que la productividad marginal del factor trabajo en Laissez-Faire con producción en el hogar, $p_{h}^{L F}$.

Demostración: Ver anexo 19.

\subsection{Equilibrio Restringido}

Para comparar los resultados en ambos modelos, se asume como punto de partida que la jornada laboral fijada por ley es la misma en el modelo con y sin producción en el hogar $\left(h_{m}^{R}=h_{m h}^{R}\right)$. En la Tabla 1 aparecen resumidas las principales variables analizadas para un valor de $\theta$ dado: 
Tabla 1. Comprobación de resultados

\begin{tabular}{ccc}
\hline \hline Variables & Sin Hogar & Con Hogar \\
\hline$w^{R}$ & $\frac{\beta \alpha(1-\sigma)\left[\phi \theta+A h_{m}^{R} \lambda\left[\frac{\theta}{d \theta^{\varepsilon}+\theta} h_{m}^{R}\right]^{\lambda-1}\right]}{[(1-\beta)+\alpha \beta(1-\sigma)] h_{m}^{R}}$ & $\frac{\beta \chi(1-\sigma)\left[\phi \theta+A h_{m}^{R} \lambda\left(\frac{h_{m}^{R} \theta}{d \theta^{\varepsilon}+\theta}\right)^{\lambda-1}\right]}{h_{m}^{R}[(1-\beta)+\beta \chi(1-\sigma)]}$ \\
$l^{R}$ & $1-h_{m}^{R}$ & $\frac{(1-\alpha)}{\gamma}\left(1-h_{m}^{R}\right)$ \\
$h_{m}^{R}$ & 0 & $\frac{\alpha \beta \eta\left(1-h_{m}^{R}\right)}{(1-\alpha)+\alpha \beta \eta}$ \\
$p^{R}$ & $A \lambda\left[\frac{\theta}{d(\theta)^{\varepsilon}+\theta} h_{m}^{R}\right]^{\lambda-1}$ & $A \lambda\left[\frac{\theta}{d\left(\theta^{R}\right)^{\varepsilon}+\theta^{R}} h_{m}^{R}\right]^{\lambda-1}$ \\
\hline
\end{tabular}

Analicemos previamente como cambian las principales variables en niveles, para posteriormente ver el impacto de la reducción de jornada. En primer lugar conviene estudiar el salario por hora de equilibrio. Dado que la participación del consumo de bienes de mercado es mayor en una economía sin actividades del hogar $(\chi<\alpha)$, los trabajadores negocian un salario más alto para hacer frente a sus necesidades de consumo, lo que implica que no incluir producción en el hogar supone sobrevalorar el nivel salarial de estado estacionario.

En segundo lugar, puede observarse que el ocio es estrictamente menor en una economía con producción en el hogar. Esto implica que si el peso de los bienes de consumo del hogar $(b)$ es suficientemente grande o $\eta$ suficientemente grande, se estaría cometiendo un sesgo importante a la hora de modelizar la reducción de jornada si se ignora las actividades del hogar.

Por último, de la tabla se desprende, que si asumieramos la misma tirantez en el mercado de trabajo, la productividad sería identica en ambos modelos.

A continuación se analiza el efecto de una reducción de jornada sobre las distintas variables. El elemento más importante que debemos considerar es como afecta una reducción de jornada a la tasa de desempleo de estado estacionario.

Proposición 8: Para un valor de $\theta$ dado, la tasa de desempleo se incrementa en una proporción mayor cuando reducimos la jornada laboral en un modelo con producción en el hogar que en un modelo sin producción en el hogar.

Demostración: Ver anexo 20.

En un modelo en el que estemos considerando las actividades que se realizan en el seno del hogar, una reducción de la jornada laboral por parte del gobierno, incrementará la tasa de desempleo de la economía en una proporción mayor a lo que lo 
haría en un modelo que no tuviese en cuenta esta actividad. Los modelos que no han introducido este elemento de la oferta, están por tanto infravalorando el efecto negativo que lleva implícito una reducción de jornada sobre la tasa de paro de la economía.

Proposición 9: Sea $\theta^{*}$ y $\theta^{* *}$ respectivamente los valores umbral superior e inferior de $\theta$ definidos por:

$$
\left[\frac{\mathrm{A} \lambda(1-\lambda) d(1-\varepsilon)}{\phi}\right]^{\lambda} h_{m}=\left(\theta^{*}\right)^{\frac{2-\varepsilon}{\lambda}}\left[1+d\left(\theta^{*}\right)^{(\varepsilon-1)}\right]
$$

$\mathrm{y}$

$$
\theta^{* *}=\left[(d+r) \varepsilon\left(\frac{(1-\beta)^{2}}{\alpha \chi \beta^{2}(1-\sigma)^{2}}-1\right)\right]^{\frac{1}{1-\varepsilon}}
$$

Para un valor $\theta^{R}$ de equilibrio dado, puede demostrarse que si $\theta^{R} \in\left[\theta^{* *}, \theta^{*}\right]$ el incremento salarial producido por una reducción de jornada será menor en un modelo con producción en el hogar.

Demostración: Ver anexo 21.

Corolario 1: A partir de la Proposiciones 8 y 9 puede demostrarse que una reducción de jornada conduce a un menor incremento del tiempo dedicado a ocio en un modelo con producción en el hogar.

\section{Calibraciones}

En esta sección se calibra el modelo con y sin producción en el hogar, para los casos de Laissez-Faire y Jornada Laboral Restringida. Se fija el valor de los parámetros de acuerdo con los usados en literatura de producción en el hogar, así como en los trabajos relacionados con modelos de emparejamientos y reducción de jornada ${ }^{24}$.

${ }^{24}$ Siguiendo el trabajo de Marimon y Zilliboti (1999) utilizamos los siguientes valores de los parámetros: $d=0,04, \lambda=0,65, \beta=0,5, \varepsilon=0,5$. Además para un valor de $u=0,033$ y tomando $\phi=0,58$, obtenemos un valor de $A=1,0$. Siguiendo el trabajo de Benhabib, J., Rogerson, R. y Wright, R. (1991) entre otros, tomamos $\sigma=0,1, \alpha=0,5, \eta=0,4$ y $a=0,5$. Por último asu$\operatorname{mimos} r=0,045$. 
Tabla 2. Equilibrio de Laissez-Faire

\begin{tabular}{lccc}
\hline & Sin Hogar & Con Hogar & Variación \\
\hline$h_{m}^{L F}$ & 0,535 & 0,344 & $-35,701 \%$ \\
$w^{L F}$ & 0,711 & 0,757 & $6,470 \%$ \\
$p^{L F}$ & 0,819 & 0,954 & $16,483 \%$ \\
$\theta^{L F}$ & 1,359 & 1,881 & $38,411 \%$ \\
$u^{L F}$ & 0,033 & 0,028 & $-15,151 \%$ \\
$c_{m}^{L F}$ & 0,381 & 0,261 & $-31,496 \%$ \\
$h_{n}^{L F}$ & $-?$ & 0,110 & - \\
$G D P^{L F}$ & 0,652 & 0,491 & $-24,693 \%$ \\
$B^{L F}$ & 0,022 & 0,029 & $3,182 \%$ \\
\hline
\end{tabular}

Como puede observarse en la Tabla 2, cuando los individuos deciden libremente la duración de la jornada, ésta resulta un $35 \%$ más pequeña en un modelo que incluye producción en el hogar. Este resultado se debe a que en un modelo con producción en el hogar los individuos obtienen utilidad, no sólo de los bienes de mercado, sino también de bienes producidos en el hogar, los cuales no dependen de las ganancias salariales.

Por otro lado en el modelo con producción en el hogar, tanto el salario por hora como la productividad son menores.

Nótese, en primer lugar, que las calibraciones recogidas en la Tabla 4 están en sintonía con la mayoría de los trabajos desarrollados hasta la fecha: una reducción de jornada genera un incremento en la tasa de desempleo, mientras que origina un incremento del salario por hora.

No obstante, en línea con lo recogido en la Proposición 8, la incorporación de actividades del hogar conduce a un efecto todavía más negativo en términos de destrucción de empleo. Además, el aumento en el salario por hora resulta menos significativo en este tipo de modelos (Proposición 9). No obstante, dado que el objetivo de este trabajo no es sólo valorar la reducción de jornada laboral como una política de creación de empleo, sino también como una política social que afecta al bienestar de los individuos, resulta necesario analizar los efectos sobre este último. Como se observa en la Tabla 4, por lo general una política de reducción de jornada tiende a incrementar el bienestar de los individuos. Si bien, el efecto resulta significativamente mayor cuando asumimos un modelo con producción en el hogar.

Así, como puede observarse, ante un reducción de jornada del $20 \%$, el aumento en el bienestar de los individuos es de alrededor de un 6.5\% en un modelo con producción en el hogar, mientras que dicho incremento no llega al 1\% cuando ignoramos las actividades dentro del hogar. 
Tabla 3. Efectos de la Reducción de Jornada sobre el Equilibrio Restringido (Niveles)

\begin{tabular}{ccccccc}
\hline & \multicolumn{2}{c}{$h_{m}^{R}=0,5$} & \multicolumn{2}{c}{$h_{m}^{R}=0,45$} & \multicolumn{2}{c}{$h_{m}^{R}=0,40$} \\
& Sin Hogar & Con Hogar & Sin Hogar & Con Hogar & Sin Hogar & Con Hogar \\
\hline$w^{R}$ & 0,726 & 0,6807 & 0,750 & 0,702 & 0,778 & 0,726 \\
$p^{R}$ & 0,839 & 0,8358 & 0,870 & 0,868 & 0,908 & 0,904 \\
$\theta^{R}$ & 1,295 & 2,4743 & 1,201 & 2,291 & 1,103 & 2,101 \\
$u^{R}$ & 0,034 & 0,0248 & 0,035 & 0,026 & 0,037 & 0,027 \\
$c_{m}^{R}$ & 0,363 & 0,340 & 0,338 & 0,316 & 0,311 & 0,290 \\
$h_{n}^{R}$ & - & 0,083 & - & 0,092 & - & 0,100 \\
$G D P^{R}$ & 0,623 & 0,627 & 0,581 & 0,585 & 0,538 & 0,542 \\
$B^{R}$ & 0,022 & 0,026 & 0,023 & 0,027 & 0,023 & 0,028 \\
\hline
\end{tabular}

Tabla 4. Efectos de la Reducción de Jornada sobre el Equilibrio Restringido (Variaciones Porcentuales)

\begin{tabular}{ccccc}
\hline $\mathrm{hRm}=0,5$ & $\begin{array}{c}-10 \% \\
\text { Sin Hogar }\end{array}$ & $\begin{array}{c}-20 \% \\
\text { Con Hogar }\end{array}$ & Sin Hogar & Con Hogar \\
\hline$w^{R}$ & 3,318 & 3,085 & 7,132 & 6,611 \\
$p^{R}$ & 3,804 & 3,793 & 8,241 & 8,196 \\
$\theta^{R}$ & $-7,275$ & $-7,416$ & $-14,798$ & $-15,083$ \\
$u^{R}$ & 3,529 & 3,629 & 7,941 & 8,468 \\
$c_{m}^{R}$ & $-7,021$ & $-7,229$ & $-14,317$ & $-14,693$ \\
$h_{n}^{R}$ & - & 10,084 & - & 20,048 \\
$G D P^{R}$ & $-6,692$ & $-6,683$ & $-13,658$ & $-13,620$ \\
$B^{R}$ & 0,893 & 3,422 & 0,892 & 6,464 \\
\hline
\end{tabular}




\section{Conclusiones}

En este trabajo se ha estudiado la reducción de la jornada laboral bajo la nueva perspectiva que supone introducir elementos de oferta de trabajo hasta ahora no analizados, en concreto el conjunto de actividades que se producen en el seno de los hogares. El objetivo de considerar la producción en el hogar, es analizar las políticas de reducción de jornada no sólo como políticas de reparto de empleo, sino como políticas sociales que tendrán implicaciones sobre el conjunto de la economía y el bienestar de los individuos.

Como se ha podido comprobar a través del modelo sin producción en el hogar, la reducción de jornada en un contexto en el que sólo se analizan algunos aspectos de la oferta (en concreto una utilidad que depende de consumo y ocio), genera resultados contundentes y bastante negativos sobre el desempleo, productividad y producción, en sintonía con los mayoría de los trabajos teóricos y empíricos desarrollados hasta la fecha.

Sin embargo la inclusión de la producción en el hogar, hace darnos cuenta que estos resultados son todavía más negativos de los descritos hasta la fecha y que en la mayoría de los casos se ha estado infravalorando el efecto negativo de una reducción de jornada.

En primer lugar podemos comprobar cómo, a pesar de que la reducción de la jornada incrementa el tiempo dedicado a actividades productivas en los hogares, el efecto sobre el desempleo, y por ende sobre la producción, es muy negativo. En este nuevo marco la reducción de jornada se vislumbra como una medida que destruye más empleo del previsto. Además la inclusión de la producción en el hogar provoca que la valoración que se hace de la productividad marginal del factor trabajo sea inferior a la que se había realizado anteriormente en el modelo sin producción en el hogar, lo que orgina un descenso de los niveles de producción.

En segundo lugar, tal y como se desprende del modelo, si los individuos pueden dedicar tiempo a actividades productivas fuera del mercado, querrán tener jornadas más cortas aunque ello suponga un recorte en su salario. Claramente, el hecho de no considerar el tiempo que los individuos dedican a producir en el hogar, genera una sobreestimación del impacto de la reducción de jornada sobre el tiempo dedicado al ocio.

En lo concerniente al salario, la reducción de la jornada de trabajo provoca una reducción salarial mayor de la que se estaba considerando en los modelos anteriormente desarrollados. Si bien el salario por hora crece, no así las ganancias salariales, que son inferiores a lo que se estaba considerando hasta la fecha. 
Una de las aportaciones más importantes de este trabajo es analizar el impacto de una política de reducción de jornada sobre el bienestar de los individuos. Tal y como se ha visto, la reducción de jornada incrementa el bienestar de los individuos en una proporción mayor que en un modelo que no incorpora las actividades de producción en el hogar.

El presente trabajo nos lleva a una conclusión clara, podemos desterrar las políticas de reducción de jornada como políticas de creación de empleo, si bien pueden considerarse políticas sociales, que permiten una mayor conciliación de la vida familiar y laboral, incrementando el bienestar de los individuos de la economía.

Existen muchos aspectos y extensiones para futuras investigaciones. Por ejemplo, relajar el supuesto de perfecta sustitución horas-hombre, para estudiar el caso en el que las últimas horas sean menos productivas.

De igual modo resultaría interesante introducir mayor heterogeneidad en el modelo, asumiendo distintos tipos de trabajadores o bien distintos tipos de sectores empresariales a la hora de analizar el impacto de una reducción de jornada.

Otra posible fuente de investigación radicaría en asumir la existencia de capital endógeno, sobre el que las empresas tienen que decidir su nivel en cada momento, y el efecto que sobre esta decisión tendría una reducción de jornada.

\section{REFERENCIAS}

[1] Andrews, M.J., Schank, T. y Simmons, R. (2005) «Does worksharing work? Some empirical evidence from the IAB-establishment panel», Scottish Journal of Political Economy, 52(2), 141-176.

[2] Benhabib, J., Rogerson, R. y Wright, R. (1990). «Homework in Macroeconomics I: Basic Theory», NBER, Working Paper Series No. 3344.

[3] Benhabib, J., Rogerson, R. y Wright, R. (1990) «Homework in Macroeconomics II: Aggregate Fluctuations», NBER, Working Paper Series No. 3344.

[4] Benhabib, J., Rogerson, R. yWright, R. (1991) «Homework in Macroeconomics: Household Production and Aggregate Fluctuations», Journal of Political Economy, 99(6), 11661187.

[5] BiLs, M. (1987) «The Cyclical Behavior of Marginal Cost and Price», The American Economic Review, 77(5), 838-855.

[6] Boоth, A. y Schiantarelli, F. (1986) «The Employment Effects of a Shorter Working Week», Economica, 54, 237-248.

[7] Calmorfs, L. (1985) «Work Sharing, Employment and Wages», European Economic Review, 27, 293-309. 
[8] Calmorfs, L. y Hoel, M. (1988) «Work Sharing and Overtime», Scandinavian Journal of Economics, 90(1), 45-62.

[9] Calmorfs, L. y Hoel, M. (1989) «Work Sharing, Employment and Shiftwork», Oxford Economic Papers, 41, 758-773.

[10] Cooley, T.F. (1995). Frontiers of Business Cycle Research. Princeton, N.J.: Princeton University Press.

[11] Costa, D. (1998) «The Unequal Work Day: A Long-Term View», The American Economic Review, 88(2), 330-334.

[12] Crepón, B. y Kramarz, F. (2002) «Employed 40 hours or not-employed 39: Lessons from 1982 mandatory reduction of the workweek», IZA Discussion Paper Series No. 416.

[13] Dolado, J. y Gómez, R. (1995) «Creación y Destrucción de Empleo en el Sector Manufacturero Español: Un Análisis Descriptivo». Investigaciones Económicas, 19(3), 371-393.

[14] Fitzgerald, J.T. (1996) «Work Schedules, Wages and Employment in a General Equilibrium Model with Team Production»,Working Paper 9613, Federal Reserve Bank of Cleveland.

[15] Fitzgerald, J.T. (1996) «Reducing Working Hours: American Workers' Salvation», Economic Commentary, Federal Reserve Bank of Cleveland.

[16] Fitzgerald, J.T. (1998) «Reducing Working Hours: A General Equilibrium Analysis», Working Paper 9801, Federal Reserve Bank of Cleveland.

[17] Fitzgerald, J.T. (1998) «Reducing Working Hours», Economic Review, 32(4). Federal Reserve Bank of Cleveland.

[18] Greenwood, J., Rogerson, R. y Wright, R. (1993) «Putting Home Economics into Macroeconomics», Federal Reserve Bank of Minneapolis, Discussion Paper V.17.No.3.

[19] Greenwood, J., Rogerson, R. y Wright, R. (1995) «Household Production in Real Business Cycle Theory», Frontiers of Business Cycle Research. Princeton, N.J.: Princeton University Press.

[20] Gronau, R. y Hamermesh, D. (2001) «The demand for variety: A household production perspective», NBER, Working Paper Series No. 8509.

[21] Hamermesh, D. (1998) «Work or Leisure: A Changing Decision?», The American Economic Review, 88(2), 321-325.

[22] Hansen, G.D. (1985) «Indivisible Labor and the Business Cycle», Journal of Monetary Economics, 16, 309-27.

[23] HaRT, R. (1984) «WorkSharing and Factor Prices», European Economic Review, 24, 165 188.

[24] HoEL, M. (1985) «Employment and Allocation Effects of Reducing the Length of the Workday», Economica, 53, 75-85.

[25] Hoel, M. y Vale, B. (1986) «Effects on Unemployment of Reduced Working Time in an Economy where Firms SetWages», European Economic Review, 30, 1097-1104.

[26] Hornstein, A. y Prescott, E.C. (1993). The Firm and the Plant in General Equilibrium Theory, 394-410. Academic Press. 
[27] Hunt, J. (1996) «The Response of wages and actual hours worked to the reductions of standard hours», NBER, Working Paper Series No. 5716.

[28] Hunt, J. (1999) «Has work-sharing worked in Germany?», The Quarterly Journal of Economics, 114(1), 117-148.

[29] JefFerys, S. (2000) «A «Copernican Revolution» in French industrial relations: Are the times a «Changing»?», British Journal of Industrial Relations, 38, 241-260.

[30] Kapteyn, A., Kalwi, A. y Zaidi, A. (2000) «The Myth of Worksharing», CENTER, Tilburg University, Working Paper No. 23.

[31] Kapteyn, A., Kalwis, A.y ZAIDI, A. (2004) «The Myth of Worksharing», Labour Economics, 11(3), 293-313.

[32] Kydland, F.E. (1995) «Business Cycle and Aggregate Labor Market Fluctuations», Frontiers of Business Cycle Research. Princeton, N.J.: Princeton University Press.

[33] LesLie, D. (1987) «Motivating Wage Structures», European Economic Review, 31, 1267-1283.

[34] Marimon, R. y Zilibotti, F. (1999) «Employment and Distributional Effects of Restricting Working Time», Working Paper No. 99/19. EUI.

[35] Marimon, R. y Zilibotтi, F. (2000) «Employment and Distributional Effects of Restricting Working Time», European Economic Review, 44, 1291-1326.

[36] Merz, M. (1995) «Search in the labor Market and the Real Business Cycle», Journal of Monetary Economics, 36, 269-300.

[37] Mortensen, D. y Pissarides, C. (1994) «Job Creation and Job Destruction in the Theory of Un employment», Review of Economic Studies, 61, 397.

[38] Mortensen, D. y Pissarides, C. (1999) «New Developments in Models of Search in the Labor Market». Handbook of Labor Economics. Vol. 3B. Chap.39.

[39] Moselle, B. (1996) «Efficiency Wages and the Hours/Unemployment Trade-Off», Northwestern University, Center for Mathematical Studies in Economics and Management Sciencie, Discussion Paper No.1153.

[40] Nickell, S. (1978) «Fixed Costs, Employment and Labour Demand Over the Cycle», Economica, 45, 329-345.

[41] Nosal, E., Rogerson, R. y Wright, R. (1992) «The Role of Household Production in Models of Involuntary Unemployment and Underemployment», Canadian Journal of Economics, 25(3), 507-520.

[42] Ortega, J. (2000) «Work-Sharing, Working Time Rigidities, and Efficiency», Mimeo, GREMAQ-IDEI.

[43] Osuna, V., Dolado, J.J. y Ríos-Rull, J.V. (2000) «Regulación en el Mercado Laboral y Mecanismos de ajustes del Empleo en las Empresas: Implicaciones Macroeconómicas», Tesis Doctoral Universidad Carlos III de Madrid.

[44] Osuna, V y Ríos-Rull, J.V. (2003) «Implmenting the 35 hour workweek by means of overtime taxation», Review of Economic Dynamics, 6, 179-206.

[45] Parente, S., Rogerson, R. y Wright, R. (1999) «Household Production and Development», Economic Review, Federal Reserve Bank of MinneapolisCleveland Q III, 21-35. 
[46] Pissarides, C. (1990). Equilibrium Unemployment. Oxford: Blackwell.

[47] Pissarides, C. (2000). Equilibrium Unemployment. Oxford: Blackwell.

[48] Pissarides, C. (1994) «Search Unemployment with On-the Job Search», Review of Economic Studies 61,457-475.

[49] Ríos-Rull, J.V. (1993) «Working in the Market, Working at Home, and the Acquision of Skills: A General-Equilibrium Approach», The American Economic Review, 83(4), 893 907.

[50] Rocheteau, G. (2000) «Working Time Regulation in a Search Economy with Worker Moral Hazard», HEC-DEEP, University of Lausanne, Working Paper.

[51] De Rongé, A. y Molitor, M (1991) «Working time in transition: the political economy of working hours in industrial nations», The Reduction of working hours in Belgium: Stakes and confrontations, in: K. Hinrichs, W.K. Roche and C. Siranni (eds.), 149-169.

[52] Rogerson, R. (1988) «Indivisible Labour Lotteries and Equilibrium», Journal of Monetary Economics, 21, 3-16. 


\section{Anexos}

\section{Anexo 1}

En primer lugar se maximiza (15) respecto al número de horas trabajadas $\left(h_{m}\right)$ :

$$
-\beta\left(J_{i}-V\right) u_{h m}^{e}=(1-\beta)\left(W_{i}-U\right)[p-w]
$$

de las condiciones (24)-(27), asumiendo la condición $V=0$, obtenemos:

$$
\begin{gathered}
{[W-U]=\frac{u^{e}-u^{u}}{r+d+\theta^{1-\varepsilon}}} \\
{[J-V]=\phi \theta^{\varepsilon}}
\end{gathered}
$$

sustituyendo (76), (77) y (20) en (75) obtengo (28).

En segundo lugar Maximizando la ecuación (15) respecto al salario (w) se obtiene:

$$
\beta\left(J_{i}-V\right) u_{w}^{e}=(1-\beta)\left(W_{i}-U\right) h_{m}
$$

Por último, sustituyendo (76), (77) y (20) en (78) se obtiene (29).

\section{Anexo 2}

A partir de (22) se obtienen las siguientes expresiones para $u_{h m}^{e}$ y $u_{w}^{e}$ :

$$
\begin{gathered}
u_{h m}^{e}\left(w, h_{m}\right)=\left[\left(w h_{m}\right)^{\alpha}\left(1-h_{m}\right) 1-\alpha\right]^{-\sigma} \\
{\left[\alpha\left(w h_{m}\right)^{\alpha-1} w\left(1-h_{m}\right) 1^{-\alpha}-(1-\alpha)\left(w h_{m}\right)^{\alpha}\left(1-h_{m}\right)^{-\alpha}\right]} \\
u_{w}^{e}\left(w, h_{m}\right)=\left[\left(w h_{m}\right)^{\alpha}\left(1-h_{m}\right) 1^{-\alpha}\right]^{-\sigma} \alpha\left(w h_{m}\right)^{\alpha-1} h_{m}\left(1-h_{m}\right)^{1-\alpha}
\end{gathered}
$$

Sustituyendo (79) y (80) en (28) y (29), obtenemos (30) y (31) respectivamente. 


\section{Anexo 3}

Tomando (24) y (26), conocidos los valores de la función de utilidad para un individuo empleado y uno desempleado dadas por (22) y (23) respectivamente y conocido (76), podemos expresar (37) como:

$$
B^{L F}=n r\left[\frac{\left(r+\left(\theta^{L F}\right)^{1-\varepsilon}\right) u^{e}}{r+d+\left(\theta^{L F}\right)^{1-\varepsilon}}\right]+(1-n)\left[\frac{\left.\left(\theta^{L F}\right)^{1-\varepsilon}\right) u^{e}}{r+d+\left(\theta^{L F}\right)^{1-\varepsilon}}\right]
$$

conocido el valor de $\mathrm{n}$ en equilibrio dado por la condición de vaciado de mercado (2), sustituyendo en (81) llegamos a (38).

\section{Anexo 4}

Maximizando (39) respecto a w obtenemos:

$$
\beta\left(\mathrm{J}_{\mathrm{i}}-V\right) u_{w}^{e}=(1-\beta)\left(W_{i}-U\right) h_{m}^{R}
$$

Procediendo de forma similar al Anexo 1, llegamos a:

$$
\frac{\beta u_{w}^{e}}{u^{e}-u^{u}}=\frac{(1-\beta) h_{m}^{R}}{[p-w] h_{m}^{R}+\phi \theta^{R}}
$$

sustituyendo (22), (23) y (80) en (83) obtenemos (40). 


\section{Anexo 5}

Conocida la ecuación (44), podemos comprobar que:

$$
\begin{gathered}
\frac{\partial \Phi^{R}\left(\theta^{R}, h_{m}^{R}\right)}{\partial \theta^{R}}=\Phi \theta_{\theta^{R}}^{R}\left(\theta^{R}, h_{m}^{R}\right)=-(d+r) \in\left(\theta^{R}\right)^{\varepsilon-1} \\
A d(1-\beta)(1-\varepsilon)\left(\theta^{R}\right)^{\varepsilon-2} \lambda(1-\lambda)\left[\frac{h_{m}^{R} \theta^{R}}{d\left(\theta^{R}\right)^{\varepsilon}+\theta^{R}}\right]^{\lambda}+\phi \alpha \beta(1-\sigma) \\
(1-\beta)+\alpha \beta(1-\sigma) \\
\frac{\partial \Phi^{R}\left(\theta^{R}, h_{m}^{R}\right)}{\partial h_{m}^{R}}=\frac{A(1-\beta) \lambda\left[\frac{h_{m}^{R} \theta^{R}}{d\left(\theta^{R}\right)^{\varepsilon}+\theta^{R}}\right]^{\lambda-1}}{(1-\beta)+\alpha \beta(1-\sigma)}
\end{gathered}
$$

Por tanto, el efecto de la reducción de jornada sobre $\theta^{R}$ viene dado por:

$$
\begin{gathered}
\frac{d \theta^{R}}{d h_{m}^{R}}=\frac{\Phi_{h_{m}^{R}}\left(\theta^{R}, h_{m}^{R}\right)}{\Phi \theta_{\theta^{R}}^{R}\left(\theta^{R}, h_{m}^{R}\right)}= \\
\frac{A(1-\beta) \lambda^{2}\left[\frac{h_{m}^{R} \theta^{R}}{d\left(\theta^{R}\right)^{\varepsilon}+\theta^{R}}\right]^{\lambda-1}}{\phi(d+r) \varepsilon\left(\theta^{R}\right)^{\varepsilon-1}[(1-\beta)+\alpha \beta(1-\sigma)]+\operatorname{Ad}(1-\beta)(1-\varepsilon)\left(\theta^{R}\right)^{\varepsilon-2} \lambda(1-\lambda)\left[\frac{h_{m}^{R} \theta^{R}}{d\left(\theta^{R}\right)^{\varepsilon}+\theta^{R}}\right]^{\lambda}+\phi \alpha \beta(1-\sigma)}
\end{gathered}
$$

Operando en la ecuación (86) llegamos a:

$$
\frac{d \theta^{R}}{d h_{m}^{R}}=\frac{\Phi_{h_{m}^{R}}\left(\theta^{R}, h_{m}^{R}\right)}{\Phi_{\theta^{R}}\left(\theta^{R}, h_{m}^{R}\right)}>0
$$

De la ecuación (2) se obtiene que la tasa de desempleo de equilibrio es

$$
u^{R}\left(\theta^{R}\right)=\frac{d}{d+\theta^{1-\varepsilon}}
$$

Por tanto, si se reduce $\theta^{R}$ entonces $u^{R}$ aumentará. 


\section{Anexo 6}

De igual modo al anexo 5, tomando la ecuación (42) conocemos el efecto de la reducción en la productividad del factor trabajo:

$$
\begin{gathered}
\frac{d p^{R}}{d h_{m}^{R}}=-A \lambda(1-\lambda)\left[\frac{\theta^{R}}{d\left(\theta^{R}\right)^{2}+\theta^{R}} h_{m}^{R}\right]^{\lambda-2} \\
{\left[\frac{\theta^{R}}{d\left(\theta^{R}\right)^{2}+\theta^{R}}+\frac{d(1-\varepsilon)\left(\theta^{R}\right)^{\varepsilon}}{\left[d\left(\theta^{R}\right)^{2}+\theta^{R}\right]^{2}} h_{m}^{R} \frac{d \theta^{R}}{d h_{m}^{R}}\right]}
\end{gathered}
$$

\section{Anexo 7}

Conocida (45) podemos determinar el efecto de la política en el salario por hora:

$$
\left.\begin{array}{c}
\frac{d w^{R}}{d h_{m}^{R}}=\frac{-(1-\sigma) \alpha \beta}{\left(h_{m}^{R}\right)^{2}[(1-\beta)+\alpha \beta(1-\sigma)]} \\
\phi \theta^{R}+A \lambda(1-\lambda)\left[\frac{\theta^{R}}{d\left(\theta^{R}\right)^{2}+\theta^{R}} h_{m}^{R}\right]^{\lambda}\left(d\left(\theta^{R}\right)^{\varepsilon-2}+1\right) \\
+h_{m}^{R}\left[\frac{d \theta^{R}}{d h_{m}^{R}}\right]\left(A \lambda(1-\lambda)\left[\frac{\theta^{R}}{d\left(\theta^{R}\right)^{2}+\theta^{R}} h_{m}^{R}\right]^{\lambda}\left(d(1-\varepsilon)\left(\theta^{R}\right)^{\varepsilon-2}-\phi\right)\right.
\end{array}\right\}
$$

donde podemos ver que $\frac{d w^{R}}{d h_{m}^{R}}<0$ si:

$$
\left[\left(A \lambda(1-\lambda)\left[\frac{\theta^{R}}{d\left(\theta^{R}\right)^{2}+\theta^{R}} h_{m}^{R}\right]^{\lambda} d(1-\varepsilon)\left(\theta^{R}\right)^{\varepsilon-2}-\phi\right] \geq 0\right.
$$

en otro caso el resultado es ambiguo. Reescribimos (90): 


$$
\left[\frac{(A \lambda(1-\lambda) d(1-\varepsilon)}{\phi}\right]^{\lambda} h_{m} \leq\left(\theta^{*}\right)^{\frac{2-\varepsilon}{\lambda}}\left[1+d\left(\theta^{*}\right)^{(\varepsilon-1)}\right]
$$

definiendo $\theta^{*}$ como el valor que satisface:

$$
\left[\frac{(A \lambda(1-\lambda) d(1-\varepsilon)}{\phi}\right]^{\lambda} h_{m}=\left(\theta^{*}\right)^{\frac{2-\varepsilon}{\lambda}}\left[1+d\left(\theta^{*}\right)^{(\varepsilon-1)}\right]
$$

\section{Anexo 8}

Conocido el valor de $B^{R}\left(=r n u^{e}\left(h_{m}^{R}, w^{R}\right)\right)$, el impacto de la reducción de jornada sobre el Bienestar de los individuos vendrá dado por:

$$
\begin{gathered}
\frac{d B^{R}}{d h_{m}^{R}}= \\
\frac{r}{1-\sigma}\left[\frac{(1-\varepsilon)\left(\theta^{R}\right)^{\varepsilon-2}}{d\left(\theta^{R}\right)^{\varepsilon-2}+1} \frac{d \theta^{R}}{d h^{R}}\right]\left[\left(w^{R} h_{m}^{R}\right)^{\alpha}\left(1-h_{m}^{R}\right)^{1-\alpha}\right]^{1-\sigma} \\
+r\left[\frac{1}{d\left(\theta^{R}\right)^{\varepsilon-2}+1}\right]\left[\left(w^{R} h_{m}^{R}\right)^{\alpha}\left(1-h_{m}^{R}\right)^{1-\alpha}\right]^{1-\sigma}\left\{\alpha\left[\frac{d w^{R}}{d h_{m}^{R}} \frac{1}{w^{R}}+\frac{1}{h_{m}^{R}}\right]-\frac{(1-\sigma)}{1-h_{m}^{R}}\right\}
\end{gathered}
$$

\section{Anexo 9}

Seguimos el mismo procedimiento del Anexo 1. Resolvemos el programa de negociación de Laissez-Faire dado por (15). Lo que nos permite llegar a las condiciones de primer orden:

En primer lugar se maximiza la ecuación (15) respecto al número de horas trabajadas $\left(h_{m h}\right)$ :

$$
-\beta\left(J_{i}-V\right) v_{h_{m h}}^{e}=(1-\beta)\left(W_{i}-U\right)\left[p_{h}-w_{h}\right]
$$

utilizando las condiciones (25), (27), (48) y (49), asumiendo la condición $V=0$, obtenemos (76) y (77) pero para la nueva función de utilidad con producción en el hogar. 
Sustituyendo (76),(77) y (20) en (94) obtengo (58).

En segundo lugar, maximizando la ecuación (15) respecto al salario $\left(w_{h}\right)$ :

$$
\beta\left(J_{i}-V\right) v_{w h}^{e}=(1-\beta)\left(W_{i}-U\right) h_{m h}
$$

sustituyendo (76),(77) y (20) en (95) obtengo (59).

\section{Anexo 10}

A partir de la especificación de la función de utilidad indirecta, dada por la ecuación (56), podemos obtener las derivadas parciales:

$$
\begin{gathered}
v_{w h}^{e}\left(w_{h}, h_{m h}\right)=\left[\left(w_{h} h_{m h}\right)^{\chi}\left(1-h_{m h}\right)\right]^{-\sigma} \\
{\left[\chi\left(w_{h} h_{m h}\right)^{\chi-1} w_{h}\left(1-h_{m h}\right)^{\gamma}-\gamma\left(w_{h} h_{m h}\right)^{\chi}\left(1-h_{m h}\right)^{\gamma-1}\right]} \\
v_{w h}^{e}\left(w_{h}, h_{m h}\right)=\left[\left(w_{h} h_{m h}\right)^{\chi}\left(1-h_{m h}\right)\right]^{-\sigma} \chi\left(w_{h} h_{m h}\right)^{\chi-1} h_{m h}\left(1-h_{m h}\right)
\end{gathered}
$$

sustituyo (96) y (97) en (58) y (59), se obtiene:

$$
\begin{gathered}
h_{m h}^{L F}:-\beta(1-\sigma)\left\{\frac{c\left(1-h_{m h}^{L F}\right)-\gamma h_{m h}^{L F}}{\left(1-h_{m h}^{L F}\right) h_{m h}^{L F}}\right\}=\frac{(1-\beta)\left[p_{h}^{L F}-w_{m h}^{L F}\right]}{\left[p_{h}^{L F}-w_{m h}^{L F}\right]+\phi \theta_{h}^{L F}} \\
w_{h}^{L F}: \frac{\beta(1-\sigma) \chi}{w_{h}^{L F}}=\frac{(1-\beta) h_{m h}^{L F}}{\left[p_{h}^{L F}-w_{m h}^{L F}\right]+\phi \theta_{h}^{L F}}
\end{gathered}
$$

lo que nos permite llegar a (60) y (61). 


$$
\begin{gathered}
\text { Anexo 11 } \\
\frac{1}{\Delta}=\frac{(d+r)\left(\theta_{h}^{L F}\right)^{\varepsilon}\left(\theta_{h}^{L F}+d\left(\theta_{h}^{L F}\right)^{\varepsilon}\right)}{\theta_{h}^{L F}\left\{\beta \theta_{h}^{L F}(1-\sigma) \chi+(d+r)\left(\theta_{h}^{L F}\right)^{\varepsilon}[1-\beta+\beta \chi(1-\sigma)]\right\}} \\
+\frac{\beta \gamma\left[\theta_{h}{ }^{L F}+d\left(\theta_{h}{ }^{L F}\right)^{\varepsilon}\right]\left[\theta_{h}^{L F}+(d+r)\left(\theta_{h}^{L F}\right)^{\varepsilon}\right](1-\sigma)+\beta\left(\theta_{h}^{L F}\right)^{2}(1-\sigma) \chi}{\theta_{h}^{L F}\left\{\beta \theta_{h}^{L F}(1-\sigma) \chi+(d+r)\left(\theta_{h}^{L F}\right)^{\varepsilon}[1-\beta+\beta \chi(1-\sigma)]\right\}} \\
+\frac{\beta d(d+r)\left(\theta_{h}^{L F}\right)^{2 \varepsilon}[1+(1-\sigma) \chi]}{\theta_{h}^{L F}\left\{\beta \theta_{h}^{L F}(1-\sigma) \chi+(d+r)\left(\theta_{h}^{L F}\right)^{\varepsilon}[1-\beta+\beta \chi(1-\sigma)]\right\}} \\
+\frac{\beta\left(\theta_{h}^{L F}\right)^{1+\varepsilon}(d+r+(2 d+r)(1-\sigma) \chi}{\theta_{h}^{L F}\left\{\beta \theta_{h}^{L F}(1-\sigma) \chi+(d+r)\left(\theta_{h}^{L F}\right)^{\varepsilon}[1-\beta+\beta \chi(1-\sigma)]\right\}}
\end{gathered}
$$

\section{Anexo 12}

Maximizando (39) respecto a $w_{h}$ obtenemos:

$$
\beta\left(J_{i}-V\right) v_{w h}^{e}=(1-\beta)\left(W_{i}-U\right) h_{m h}^{R}
$$

Procediendo de forma similar al Anexo 1, llegamos a:

$$
\frac{\beta v_{w h}^{e}}{v^{e}-v^{u}}=\frac{(1-\beta) h_{m h}^{R}}{\left[p_{h}^{R}-w_{h}^{R}\right] h_{m h}^{R}+\phi \theta_{h}^{R}}
$$

sustituyendo (56), (57) y (97) en (102) obtenemos (67).

\section{Anexo 13}

$\operatorname{Si}(1-\alpha)>\alpha \beta \eta \Rightarrow \frac{-(1-\alpha)}{(1-\alpha)+\alpha \beta \eta}<\frac{-\alpha \beta \eta}{(1-\alpha)+\alpha \beta \eta} \Rightarrow \frac{d l_{h}}{d h_{m h}}<\frac{d h_{m h}}{d h_{m h}}<0$. 


\section{Anexo 14}

Conocido (70), podemos calcular:

$$
\begin{gathered}
\frac{\partial \Psi\left(\theta_{h}^{R}, h_{m h}^{R}\right)}{\partial \theta_{h}^{R}}=-\phi(d+r) \varepsilon\left(\theta_{h}^{R}\right)^{\varepsilon-1} \\
A d(1-\beta)(1-\varepsilon)\left(\theta_{h}^{R}\right)^{\varepsilon-2} \lambda(1-\lambda)\left(\frac{\theta_{h}^{R} h_{m h}^{R}}{\theta_{h}^{R}+d\left(\theta_{h}^{R}\right)^{\varepsilon}}\right)^{\lambda}+\phi \chi \beta(1-\sigma) \\
(1-\beta)+\chi \beta(1-\sigma) \\
\frac{\partial \Psi\left(\theta_{h}^{R}, h_{m h}^{R}\right)}{\partial h_{m h}^{R}}=\frac{A(1-\beta) \lambda^{2}\left(\frac{h_{m h}^{R} \theta_{h}^{R}}{\theta_{h}^{R}+d\left(\theta_{h}^{R}\right)^{\varepsilon}}\right)^{\lambda-1}}{(1-\beta)+\chi \beta(1-\sigma)}
\end{gathered}
$$

Con (103) y (104), sabiendo (106) obtengo:

$$
\frac{d \theta_{h}^{R}}{\partial h_{m h}^{R}}=
$$

$$
A(1-\beta) \lambda^{2}\left(\frac{h_{m h}^{R} \theta_{h}^{R}}{\theta_{h}^{R}+d\left(\theta_{h}^{R}\right)^{\varepsilon}}\right)^{\lambda-1}
$$

$A d(1-\beta)(1-\varepsilon)\left(\theta_{h}^{R}\right)^{\varepsilon-2} \lambda(1-\lambda)\left(\frac{h_{m h}^{R} \theta_{h}^{R}}{\theta_{h}^{R}+d\left(\theta_{h}^{R}\right)^{\varepsilon}}\right)^{\lambda}+\phi \chi \beta(1-\sigma)+\phi(d+r) \varepsilon\left(\theta_{h}^{R}\right)^{\varepsilon-1}[(1-\beta)+\chi \beta(1-\sigma)]$

Operando en (105) llegamos a la siguiente expresión:

$$
\frac{d \theta_{h}^{R}}{d h_{m h}^{R}}=\frac{\frac{\partial \Psi\left(\theta_{h}^{R}, h_{m h}^{R}\right)}{\partial h_{m h}^{R}}}{\frac{\partial \Psi\left(\theta_{h}^{R}, h_{m h}^{R}\right)}{\partial \theta_{h}^{R}}}>0
$$


Y por tanto, a partir de la ecuación (2), se obtiene que: $\frac{d u_{h}^{R}}{d \theta_{h}^{R}}<0$.

\section{Anexo 15}

Tomando (68) es fácil de ver que:

$$
\begin{gathered}
\frac{d p_{h}^{R}}{d h_{m h}^{R}}=-A \lambda(1-\lambda)\left[\frac{\theta_{h}^{R}}{d\left(\theta_{h}^{R}\right)^{\varepsilon}+\theta_{h}^{R}} h_{m h}^{R}\right]^{\lambda-2} \\
{\left[\frac{\theta_{h}^{R}}{d\left(\theta_{h}^{R}\right)^{\varepsilon}+\theta_{h}^{R}}+\frac{d(1-\varepsilon)\left(\theta_{h}^{R}\right)}{\left[d\left(\theta_{h}^{R}\right)^{\varepsilon}+\theta_{h}^{R}\right]^{2}} h_{m h}^{R}\left[\frac{d \theta_{h}^{R}}{d h_{m h}^{R}}\right]\right]}
\end{gathered}
$$

\section{Anexo 16}

Partimos de la ecuación (71) y estudiamos como cambia al modificarse $h_{m h}^{R}$ :

$$
\begin{gathered}
\frac{d_{w}^{R}}{d h_{m h}^{R}}=\frac{(1-\sigma) \chi \beta}{\left(h_{m h}^{R}\right)^{2}[(1-\beta)+\chi \beta(1-\sigma)]} \\
\left\{\begin{array}{c}
\phi \theta+A \lambda(1-\lambda)\left(\frac{h_{m h}^{R} \theta_{h}^{R}}{\theta_{h}^{R}+d\left(\theta_{h}^{R}\right)^{\varepsilon}}\right)^{\lambda}\left(1+d\left(\theta_{h}^{R}\right)^{\varepsilon-1}\right) \\
+h_{m h}^{R}\left[\frac{d \theta_{h}^{R}}{d h_{m h}^{R}}\right]\left[A \lambda(1-\lambda)\left(\frac{h_{m h}^{R} \theta_{h}^{R}}{\theta_{h}^{R}+d\left(\theta_{h}^{R}\right)^{\varepsilon}}\right)^{\lambda} d(1-\varepsilon)\left(\theta_{h}^{R}\right)^{\varepsilon-2}-\phi\right]
\end{array}\right\}
\end{gathered}
$$




\section{Anexo 17}

Tomando (70):

$$
\begin{gathered}
\frac{\partial \Psi\left(\theta_{h}^{R}, h_{m h}^{R}\right)}{\partial a}=\Psi a\left(\theta_{h}^{R}, h_{m h}^{R}\right)= \\
\frac{(1-\beta) \beta(1-\sigma)\left[\phi\left(\theta_{h}^{R}\right)^{2}+A \lambda h_{m h}^{R} \theta\left(\frac{h_{m h}^{R} \theta_{h}^{R}}{\theta_{h}^{R}+d\left(\theta_{h}^{R}\right)^{\varepsilon}}\right)^{\lambda-1}\right]}{\theta_{h}^{R}[(1-\beta)+\chi \beta(1-\sigma)]^{2}}\left[\frac{\partial \chi}{\partial a}\right]
\end{gathered}
$$

conociendo (103) y (109):

$$
\frac{d \theta_{h}^{R}}{d a}=-\frac{\Psi a\left(\theta_{h}^{R}, h_{m h}^{R}\right)}{\Psi \theta_{h}^{R}\left(\theta_{h}^{R}, h_{m h}^{R}\right)}=
$$

$$
\frac{\frac{(1-\beta) \beta(1-\sigma)}{[(1-\beta) \chi \beta(1-\sigma)]}\left[\phi \theta_{h}^{R}+A \lambda h_{m h}^{R}\left(\frac{h_{m h}^{R} \theta_{h}^{R}}{\theta_{h}^{R}+d\left(\theta_{h}^{R}\right)^{\varepsilon}}\right)^{\lambda-1}\right]\left[\frac{\partial \chi}{\partial a}\right]}{\phi(d+r) \varepsilon\left(\theta_{h}^{R}\right)^{\varepsilon-1}[(1-\beta)+\chi \beta(1-\sigma)]+A d(1-\beta)(1-\varepsilon)\left(\theta_{h}^{R}\right)^{\varepsilon-2} \lambda(1-\lambda)\left(\frac{h_{m h}^{R} \theta_{h}^{R}}{\theta_{h}^{R}+d\left(\theta_{h}^{R}\right)^{\varepsilon}}\right)^{\lambda}+\phi \chi \beta(1-\sigma)}
$$

\section{Anexo 18}

Conocido el valor de $h_{m}^{L F}$ dado por (35) y conocido el valor de $h_{m h}^{L F}$ (64), sabiendo que $\chi<\alpha$, entonces:

$$
h_{m}^{L F}(\theta)-h_{m h}^{L F}(\theta)=
$$

$$
\frac{(d+r)(1-\beta) \theta^{\varepsilon}+\alpha \beta\left[\theta+(d+r) \theta^{\varepsilon}\right](1-\sigma)}{\beta \theta(1-\sigma)+(d+r) \theta^{\varepsilon}[(1-\beta)+\beta(1-\sigma)]}-\frac{\beta \theta \chi(1-\sigma)+(d+r) \theta^{\varepsilon}[(1-\beta)+\beta \chi(1-\sigma)]}{\beta \theta(\chi-\gamma)(1-\sigma)+(d+r) \theta^{\varepsilon}[(1-\beta)+\beta(\chi-\gamma)(1-\sigma)]}
$$


si $\alpha(\chi+\gamma)-\chi>0$, entonces:

$$
h_{m}^{L F}(\theta)-h_{m h}^{L F}(\theta)>0
$$

\section{Anexo 19}

Comparando (32) y (62), vemos que la única diferencia es la que viene determinada por $(\gamma+\chi)$. Tal y como hemos definido anteriormente $\gamma=\alpha \beta \eta+(1-\alpha) \mathrm{y}$ $\chi=a \alpha$, por tanto: $\gamma+\chi=\alpha \beta \eta+(1-\alpha)+a \alpha=1+\alpha[a+b \eta-1]$.

Supongamos que $(\gamma+\chi)>1 \Rightarrow \alpha[a+b \eta-1]>0$, dado que $0<\alpha, b, \eta, a<1$ y además sabiendo que $a+b=1$, entonces: $a+b \eta-1>0 \Rightarrow b \eta>b$ que es imposible.

Queda demostrado por tanto: $(\gamma+\chi)<1$.

\section{Anexo 20}

Tomando (86) y (105), vemos que:

$$
\begin{gathered}
\frac{d \theta^{R}}{\frac{d h_{m}^{R}}{d \theta^{R}}}= \\
\frac{d h_{m h}^{R}}{A d(1-\beta)(1-\varepsilon) \theta^{\varepsilon-2} \lambda(1-\lambda)\left(\frac{h_{m h}^{R} \theta}{\theta+d \theta^{\varepsilon}}\right)^{\lambda}+\phi \chi \beta(1-\sigma)+\phi(d+r) \varepsilon \theta^{\varepsilon-1}[(1-\beta)+\chi \beta(1-\sigma)]} \\
\operatorname{Ad}(1-\beta)(1-\varepsilon) \theta^{\varepsilon-2} \lambda(1-\lambda)\left(\frac{h_{m}^{R} \theta}{d \theta^{\varepsilon}+\theta}\right)^{\lambda}+\phi \alpha \beta(1-\sigma)+\phi(d+r) \varepsilon \theta^{\varepsilon-1}[(1-\beta)+\alpha \beta(1-\sigma)]
\end{gathered}
$$

nuevamente, dado que $\chi<\alpha$ entonces: 


$$
\frac{\frac{d \theta^{R}}{d h_{m}^{R}}}{\frac{d \theta^{R}}{d h_{m h}^{R}}}<1 \Rightarrow \frac{d \theta^{R}}{d h_{m}^{R}}<\frac{d \theta_{h}^{R}}{d h_{m h}^{R}}
$$

Sean $u^{R}$ y $u_{h}^{R}$ las tasas de desempleo en un modelo sin y con producción en el hogar respectivamente. Conocida la condición de vaciado (2), sabemos que:

$$
\begin{aligned}
& \frac{d u^{R}}{d h_{m}^{R}}=\frac{d(1-\varepsilon)\left(\theta^{R}\right)^{-\varepsilon} d \theta^{R}}{\left[d+\left(\theta^{R}\right)^{1-\varepsilon}\right]^{2}} d h_{m}^{R} \\
& \frac{d u_{h}^{R}}{d h_{m h}^{R}}=\frac{d(1-\varepsilon)\left(\theta_{h}^{R}\right)^{-\varepsilon} d \theta_{h}^{R}}{\left[d+\left(\theta_{h}^{R}\right)^{1-\varepsilon}\right]^{2}} d h_{m}^{R}
\end{aligned}
$$

por tanto, conocido (114), llegamos a:

$$
\left|\frac{d_{w}^{R}}{d h_{m h}^{R}}\right|>\left|\frac{d w_{h}^{R}}{d h_{m h}^{R}}\right|
$$

\section{Anexo 21}

Conocido los efectos de la reducción de jornada en el salario en (89) y (108), asumimos que $\theta=\theta^{R}=\theta_{h}^{R}$ tal que $\theta \leq \theta^{*}$, el cual viene definido por la ecuación (92), llegamos a:

$$
\begin{gathered}
\left|\frac{d_{w}^{R}}{d h_{m h}^{R}}\right|-\left|\frac{d w_{h}^{R}}{d h_{m h}^{R}}\right|= \\
\left\{\begin{array}{c}
{\left[\frac{(1-\sigma) \alpha \beta}{\left(h_{m}^{R}\right)^{2}[(1-\beta)+\alpha \beta(1-\sigma)]}-\frac{(1-\sigma) \chi \beta}{\left(h_{m}^{R}\right)^{2}[(1-\beta)+\alpha \beta(1-\sigma)]}\right]} \\
{\left[\phi \theta^{R}+A \lambda(1-\lambda)\left[\frac{\theta^{R}}{d\left(\theta^{R}\right)+\theta^{R}} h_{m}^{R}\right]^{\lambda}\left(d\left(\theta^{R}\right)^{\varepsilon-1}+1\right)\right]}
\end{array}\right\}
\end{gathered}
$$




$$
+\left\{\begin{array}{c}
{\left[A \lambda(1-\lambda)\left[\frac{\theta^{R}}{d\left(\theta^{R}\right)+\theta^{R}} h_{m}^{R}\right]^{\lambda}\left(d(1-\varepsilon)\left(\theta^{R}\right)^{\varepsilon-2}+\phi\right)\right]} \\
\left\{\frac{(1-\sigma) \alpha \beta}{h_{m}^{R}[(1-\beta)+\alpha \beta(1-\sigma)]}\left[\frac{d \theta^{R}}{d h_{m}^{R}}\right]-\frac{(1-\sigma) \chi \beta}{h_{m}^{R}[(1-\beta)+\alpha \beta(1-\sigma)]}\left[\frac{d \theta_{h}^{R}}{d \theta_{m h}^{R}}\right]\right\}
\end{array}\right.
$$

es fácil de comprobar que:

$$
\left[\frac{(1-\sigma) \alpha \beta}{\left(h_{m}^{R}\right)^{2}[(1-\beta)+\alpha \beta(1-\sigma)]}-\frac{[(1-\sigma)+\chi \beta}{\left(h_{m}^{R}\right)^{2}[(1-\beta)+\chi \beta(1-\sigma)]}\right]>0
$$

por tanto tenemos que:

$$
\begin{aligned}
& \left|\frac{d_{w}^{R}}{d h_{m h}^{R}}\right|-\left|\frac{d w_{h}^{R}}{d h_{m h}^{R}}\right|>0 \Leftrightarrow \\
& \frac{(1-\sigma) \alpha \beta}{h_{m}^{R}[(1-\beta)+\alpha \beta(1-\sigma)]}\left[\frac{d \theta^{R}}{d h_{m}^{R}}\right]-\frac{(1-\sigma) \chi \beta}{h_{m}^{R}[(1-\beta)+\alpha \beta(1-\sigma)]}\left[\frac{d \theta_{h}^{R}}{d \theta_{m h}^{R}}\right] \geq 0
\end{aligned}
$$

conocido (86), (105) y (113), nos queda:

$$
\left|\frac{d_{w}^{R}}{d h_{m h}^{R}}\right|-\left|\frac{d w_{h}^{R}}{d h_{m h}^{R}}\right|>0 \Leftrightarrow \theta \geq\left[(d+r) \varepsilon\left(\frac{(1-\beta)^{2}}{\alpha \chi \beta^{2}(1-\sigma)^{2}}\right)\right]^{\frac{1}{1-\varepsilon}}
$$

definiendo $\theta^{* *}$, tal que:

$$
\theta^{* *}=\left[(d+r) \varepsilon\left(\frac{(1-\beta)^{2}}{\alpha \chi \beta^{2}(1-\sigma)^{2}}\right)\right]^{\frac{1}{1-\varepsilon}}
$$

llegamos a que: $\theta \in\left[\theta^{* *}, \theta^{*}\right]$ para que se cumpla $\left|\frac{d_{w}^{R}}{d h_{m h}^{R}}\right|-\left|\frac{d w_{h}^{R}}{d h_{m h}^{R}}\right|>0$ 